\title{
Atmospheric Mining in the Outer Solar System: Outer Planet In-Space Bases and Moon Bases for Resource Processing
}

\author{
Bryan Palaszewski \\ NASA John H. Glenn Research Center \\ Lewis Field \\ MS 5-10 \\ Cleveland, $\mathrm{OH} 44135$ \\ (216) 977-7493 Voice \\ (216) 433-5802 FAX \\ bryan.a.palaszewski@nasa.gov \\ Fuels and Space Propellants Web Site: \\ http://www.grc.nasa.gov/WWW/Fuels-And-Space-Propellants/foctopsb.htm
}

\begin{abstract}
Atmospheric mining in the outer solar system has been investigated as a means of fuel production for high energy propulsion and power. Fusion fuels such as Helium $3(3 \mathrm{He})$ and deuterium can be wrested from the atmospheres of Uranus and Neptune and either returned to Earth or used in-situ for energy production. Helium 3 and deuterium were the primary gases of interest with hydrogen being the primary propellant for nuclear thermal solid core and gas core rocket-based atmospheric flight. A series of analyses were undertaken to investigate resource capturing aspects of atmospheric mining in the outer solar system. This included the gas capturing rate, storage options, and different methods of direct use of the captured gases. While capturing $3 \mathrm{He}$, large amounts of hydrogen and $4 \mathrm{He}$ are produced. The propulsion and transportation requirements for all of the major moons of Uranus and Neptune are presented. Analyses of orbital transfer vehicles (OTVs), landers, factories, and the issues with in-situ resource utilization (ISRU) low gravity processing factories are included. Preliminary observations are presented on near-optimal selections of moon base orbital locations, OTV power levels, and OTV and lander rendezvous points. Several artificial gravity in-space base designs and orbital sites at Uranus and Neptune and the OTV requirements to support them are also addressed.
\end{abstract}

\section{Nomenclature}

$\begin{array}{ll}3 \mathrm{He} & \text { Helium } 3 \\ \text { 4He } & \text { Helium (or Helium } 4) \\ \text { AMOSS } & \text { Atmospheric mining in the outer solar system } \\ \text { ASC } & \text { Aerospacecraft for atmospheric mining } \\ \text { CC } & \text { Closed cycle } \\ \text { delta-V } & \text { Change in velocity }(\mathrm{km} / \mathrm{s}) \\ \text { GCR } & \text { Gas core rocket } \\ \text { H2 } & \text { Hydrogen } \\ \text { He } & \text { Helium } 4 \\ \text { ISRU } & \text { In Situ Resource Utilization } \\ \text { Isp } & \text { Specific Impulse }(\mathrm{s}) \\ \text { ITV } & \text { Interplanetary transfer vehicle }\end{array}$

\footnotetext{
* Leader of Advanced Fuels, AIAA Associate Fellow
} 


\begin{tabular}{ll} 
K & Kelvin \\
kWe & Kilowatts of electric power \\
LEO & Low Earth Orbit \\
MT & Metric tons \\
MWe & Megawatt electric (power level) \\
NEP & Nuclear Electric Propulsion \\
NPP & Nuclear Pulse Propulsion \\
NTP & Nuclear Thermal Propulsion \\
NTR & Nuclear Thermal Rocket \\
OC & Open cycle \\
OTV & Orbital Transfer Vehicle \\
O2 & Oxygen \\
PPack & Physics Package for nuclear pulse propulsion \\
PPB & Parts per billion \\
\hline
\end{tabular}

\section{Atmospheric mining in the outer solar system}

Atmospheric mining of the outer solar system is one of the options for creating nuclear fuels, such as $3 \mathrm{He}$, for future fusion powered exploration vehicles or powering reactors for Earth's planetary energy. Uranus' and Neptune's atmospheres would be the primary mining sites, and robotic vehicles would wrest these gases from the hydrogen-helium gases of those planets. While preliminary estimates of the masses of the mining vehicles have been created (Refs. 1-4), additional supporting vehicles may enhance the mining scenarios. Storing the mined gases at automated bases on outer planet moons was conceived to ease the storage requirements on interplanetary transfer vehicles (that would return the cryogenic gases to Earth).

\section{Resource capturing studies}

Studies of the gas capture rate and its influence on mining time in the atmosphere were conducted. Aerospacecraft cruisers have been identified as a "best" solution for atmospheric mining (Ref. 1-12). To power these vehicles, atmospheric hydrogen gas would be liquefied and used a rocket propellant for the ascent to orbit. A gas core rocket is a likely candidate. Gaseous or liquid hydrogen would be used to power the engines during atmospheric mining operations. Helium 3 (3He) would be separated from the atmospheric hydrogen and helium (4He) captured, liquefied and stored as a payload that would be returned to orbit. A 500-kg payload of $3 \mathrm{He}$ is captured during the mining time. Table I provides the amount of $3 \mathrm{He}$ in the outer planet atmospheres.

Table I. Fraction of helium 3 in outer planet atmospheres

\begin{tabular}{|l|r|r|r|}
\hline & Uranus & Neptune \\
\hline Amount of 3He in 4He & $1.00 \mathrm{E}-04$ & $1.00 \mathrm{E}-04$ \\
\hline Amount of 4He in atmosphere & 0.152 & 0.19 \\
\hline Amount of 3He in atmosphere & $1.52 \mathrm{E}-05$ & $1.90 \mathrm{E}-05$ \\
\hline
\end{tabular}




\section{Vehicle, Mission, and Propulsion Studies}

\section{A. Moon Base Transportation and Mission Planning}

In order to illustrate the use and storage of helium 3 and deuterium, a series of analyses were conducted regarding transportation options. Several steps are needed to store the nuclear fuels. The ASC must mine the gases from the planet's atmosphere. After mining, the ASC ascends to low orbit, and then rendezvous with an orbiting tanker or orbital transfer vehicle. The OTV and ASC rendezvous at an altitude compatible with both vehicles. After the rendezvous, the OTV accepts the mined cryogenic gases from the ASC, and, the OTV begins a low thrust spiral trajectory to the storage point. In these analyses, an outer planet moon was selected as the storage point. At the moon, the OTV and lander will rendezvous in high orbit about the outer planet moon. The OTV will deliver the mined gases to a lander. The lander will refuel the OTV from hydrogen mined on the moon. The OTV will return to the rendezvous altitude to await the next ASC delivery. The lander will return to the moon with the mined gases. On the moon, the lander propulsion system will be refueled with oxygen and hydrogen from the water ice from the moon. Hydrogen for the OTV refueling will also be loaded aboard the lander. The ASC-OTV-Lander ISRU cycle continues until the needed helium 3 and deuterium are stored on the moon. References 13 to 33 provide many options for nuclear power and nuclear propulsion to support this mining.

\section{B. Mission analyses for moon basing}

In the planned mining operations, there are several transportation elements. The ASC captures the atmosphere in-situ and extracts the helium 3. The ASC then ascends to an $800 \mathrm{~km}$ orbit to rendezvous with an orbital transfer vehicle (OTV). The OTV uses low thrust nuclear electric propulsion (NEP). An $800 \mathrm{~km}$ orbit was selected as it is a high enough altitude to prevent the atmospheric drag from affecting the NEP OTV (Refs. 1-12).

The OTV then transports the helium 3 payload(s) to an orbit about a small moon of the planet. The moon is selected to have a low gravity level, so that entering and exiting the moons orbit requires a low delta-V. The moon should be an ice and rock body that can be mined for water. Water is mined on the moon to create oxygen and hydrogen to fuel high thrust chemical propulsion systems. A chemical propulsion lander then ascends from the moon to transfer the helium 3 to the moon's surface. The lander also delivers liquid hydrogen to refuel the OTV. After picking up the helium 3 payload, the lander returns to the moon's surface. After soft landing the payload on the moon, a storage facility will hold the helium 3 until needed. Possible storage times can be many years or decades, as the applications of the helium 3 are nuclear fusion fuels for interplanetary and interstellar missions, etc. The time to mine the needed fuels can literally be decades.

\section{Where do you store fuels?}

Storing fuels in low orbit above the planet is generally not desirable. The storage facility may be subject to large variations in the planet's atmosphere, and may also unintentionally reenter the planet's atmosphere. The 800 $\mathrm{km}$ altitude is above the altitude where the nominal atmospheric drag would seriously affect the NEP OTV's orbit. The ASC total delta-V capability is $20.1 \mathrm{~km} / \mathrm{s}$. The ASC delta-V includes the launch from the mining altitude at subsonic speed to a low altitude orbital velocity and then the additional ascent to the $800 \mathrm{~km}$ orbital altitude. Storing the fuels in a place with some sensible gravity is an attractive option. Many processes for mining, extracting, purifying, and larger scale production would benefit from a moon with gravity. Therefore an outer planet moon was sought as a base for facility operations and fuel storage. 


\section{Moon selection}

The moon selection for the storage of the cryogenic mined gases was based on several factors. The moon should be a water ice and rock body. The water ice will be needed to produce the propellants for the low-thrust OTV and the lander. The moon should be in an orbit away from the potential debris of the planet's ring plane. Being away from the rings minimizes the hazards that the OTV will have to traverse and avoid. The moon should have a high enough gravity level to ease or simplify the propellant production operations. The moon's gravity should be low enough to allow easy transportation onto and off of the surface with a relatively small delta-V. It is also an attractive option to select a moon far from rings, and away from the planet's radiation environment. Also, one should select small moon with minimal gravity, but enough gravity for mining operations. A cryogenic environment is excellent for long term helium 3 storage (below a temperature of $4 \mathrm{~K}$ ).

At Uranus, the moons named Miranda and Titania was selected as an example for these AMOSS OTV and lander operations. The two moons have the largest and the smallest class of delta-V missions for round trips from those moons' surfaces. Tables II and III provide the delta-V needed for these round trips for the moons of Uranus and Neptune, respectively. The delta- $\mathrm{V}$ values were computed with standard orbital mechanics equations. At Miranda, the round trip escape delta- $\mathrm{V}$ was $0.5 \mathrm{~km} / \mathrm{s}$ for the lander mission. The delta-V included the escape velocity from the moon, for each leg of the round trip, and a $20 \%$ additional delta-V for gravity losses. Titania has a spectral signature that strongly implies that it is an ice and rock body (Ref. 28 and 29). The moon's escape velocity is approximately $0.95 \mathrm{~km} / \mathrm{s}$ (including gravity losses, uncertainties, etc.). The round trip delta-V was therefore 1.9 $\mathrm{km} / \mathrm{s}$.

Tables IV and V list the round trip delta- $\mathrm{V}$ values for travel from Uranus to its moons and from Neptune to its moons, respectively. The delta- $\mathrm{V}$ values were computed with standard orbital mechanics equations. For Titania, the low-thrust round trip orbital delta- $\mathrm{V}$ would be $22.4 \mathrm{~km} / \mathrm{s}$, which includes the required plane change of 0.14 degrees. The lander that would rendezvous with an OTV would deliver $1.9 \mathrm{~km} / \mathrm{s}$ (for the round trip flight). Thus, the rendezvous could occur near the escape conditions for the moon or at the lower orbital altitude. The OTV is sized to deliver $22.4 \mathrm{~km} / \mathrm{s}$ (equal to a round trip flight to Titania from low Uranus orbit). For Neptune, the moons Thalassa and Triton were investigated. Thalassa required a round trip OTV delta-V of $9.4 \mathrm{~km} / \mathrm{s}$. The round trip lander delta-V was $80 \mathrm{~m} / \mathrm{s}$. For Triton, the round trip OTV mission required a delta-V of $26.2 \mathrm{~km} / \mathrm{s}$, and the round trip lander delta- $\mathrm{V}$ was $3.5 \mathrm{~km} / \mathrm{s}$.

Table II. Uranus Moon Lander delta-V Values

\begin{tabular}{|l|r|r|r|}
\hline Moon & $\begin{array}{l}\text { Round-trip } \\
\text { delta-V (km/s) }\end{array}$ & $\begin{array}{l}\text { delta-V } \\
\text { Capability }(\mathrm{km} / \mathrm{s})\end{array}$ & Sizing Category \\
\hline Miranda (UV) & 0.44 & 0.5 & a \\
\hline Ariel (UI) & 1.34 & 1.4 & b \\
\hline Umbriel (UII) & 1.24 & 1.3 & b \\
\hline Titania (UII) & 1.85 & 1.9 & c \\
\hline Oberon (UIV) & 1.74 & 1.8 & c \\
\hline
\end{tabular}


Table III. Neptune Moon Lander delta-V Values

\begin{tabular}{|c|c|c|c|}
\hline Moon & $\begin{array}{l}\text { Round-trip } \\
\text { delta-V (km/s) }\end{array}$ & $\begin{array}{l}\text { delta-V } \\
\text { Capability }(\mathrm{km} / \mathrm{s})\end{array}$ & Sizing Category \\
\hline Naiad (NIII) & 0.06 & 0.06 & $\mathbf{a}$ \\
\hline Thalassa (NIV) & 0.08 & 0.08 & $\mathbf{a}$ \\
\hline Despina (NV) & 0.13 & 0.14 & b \\
\hline Galatea (NVI) & 0.17 & 0.18 & b \\
\hline Larissa (NVII) & 0.19 & 0.19 & b \\
\hline \multicolumn{4}{|l|}{ S/2004 N1 } \\
\hline Proteus (NVIII) & 0.44 & 0.50 & c \\
\hline Triton & 3.49 & 3.50 & d \\
\hline Nereid & 0.37 & 0.40 & c \\
\hline
\end{tabular}

Table IV. Uranus to Moon Orbital Transfer delta-V Values

Planet to moon orbital transfer, low thrust delta-V delta-V $(\mathrm{km} / \mathrm{s}), 1$ way delta-V $(\mathrm{km} / \mathrm{s}), 2$ way delta-V capability $(\mathrm{km} / \mathrm{s})$

\begin{tabular}{|l|c|c|c|}
\hline Miranda (UV) & 8.216 & 16.432 & 16.5 \\
\hline Ariel (UI) & 9.320 & 18.640 & 18.7 \\
\hline Umbriel (UII) & 10.162 & 20.324 & 20.4 \\
\hline Titania (UIII) & 11.180 & 22.360 & 22.4 \\
\hline Oberon (UIV) & 11.675 & 23.350 & 23.4 \\
\hline
\end{tabular}

Table V. Neptune to Moon Orbital Transfer delta-V Values

\begin{tabular}{|l|c|c|c|}
\hline & \multicolumn{2}{|c|}{ Planet to moon orbital transfer, low thrust delta-V } & \\
\hline & delta-V $(\mathbf{k m} / \mathbf{s}), \mathbf{1}$ way & delta-V $(\mathbf{k m} / \mathbf{s}), 2$ way & delta-V capability $(\mathbf{k m} / \mathbf{s})$ \\
\hline Naiad (NIII) & 4.808 & 9.616 & 9.7 \\
\hline Thalassa (NIV) & 4.675 & 9.350 & 9.4 \\
\hline Despina (NV) & 4.950 & 9.900 & 9.9 \\
\hline Galatea (NVI) & 5.860 & 11.720 & 11.8 \\
\hline Larissa (NVII) & 6.720 & 13.440 & 13.5 \\
\hline S/2004 N1 & too small & & \\
\hline Proteus (NVIII) & 8.736 & 17.472 & 17.5 \\
\hline Triton & 13.072 & 26.144 & 26.2 \\
\hline Nereid & 15.269 & 30.538 & 30.6 \\
\hline
\end{tabular}

\section{E. Factory design issues}

One or more factories will be needed in the transportation system. With AMOSS, there is a gas separation and liquefaction system or factory on the ASC. Also needed will be one or two factories on the moon for payload storage. One factory will create oxygen and hydrogen for moon lander propellants. Another may be needed to 
process the helium 3 (and other fusion fuels such as deuterium). The factory sizing was estimated by breaking down the factory into the planned components.

The gravity levels of the moons will be one of the critical issues. Many of the outer planet moons have gravity levels far below $0.1 \mathrm{G}$ (at Earth), leading to the strong suggestion for the need for an artificial gravity platform for processing. Processing in orbit about the moon may make the best sense for the nuclear fuels processing. The factory mass for nuclear fuel elements (physics packages, PPack) or nuclear fusion pellets was also estimated. There are large uncertainties in the number of required processes, and complexities associated with low gravity processing of nuclear materials. Water from the moon surface would be separated on the surface, perhaps with centrifuges to separate the rock elements and the water ices.

The most important processes may indeed be the basing and emplacement of the propellant and PPack factories. The safe and reliable operation of the factory for decades will be necessary. Also, purification of the water and other gases will be critical. Typically, water is full of salts, as with the Earth's oceans. The same is true for any interplanetary moons' water supplies (Ref. 34 and 35). Using the propellants in chemical or electric propulsion systems and in the operations of the factories will necessitate their purification. :

Uranus' moons have a higher gravity level than many of the moons of Neptune. At Neptune, Proteus and Triton are the only moons with a comparable or higher gravity level. A gravity level of $10^{\wedge}-3$ Gs is comparable to the transient disturbance accelerations on the ISS, and this acceleration level is likely for any other space station (thruster firing, etc., Ref. 36). It is therefore likely that an orbiting station may be a good and/or bad location for a propellant ISRU processing center. It may be bad in that the orbiting platform will have transients that will disturb any microgravity process.

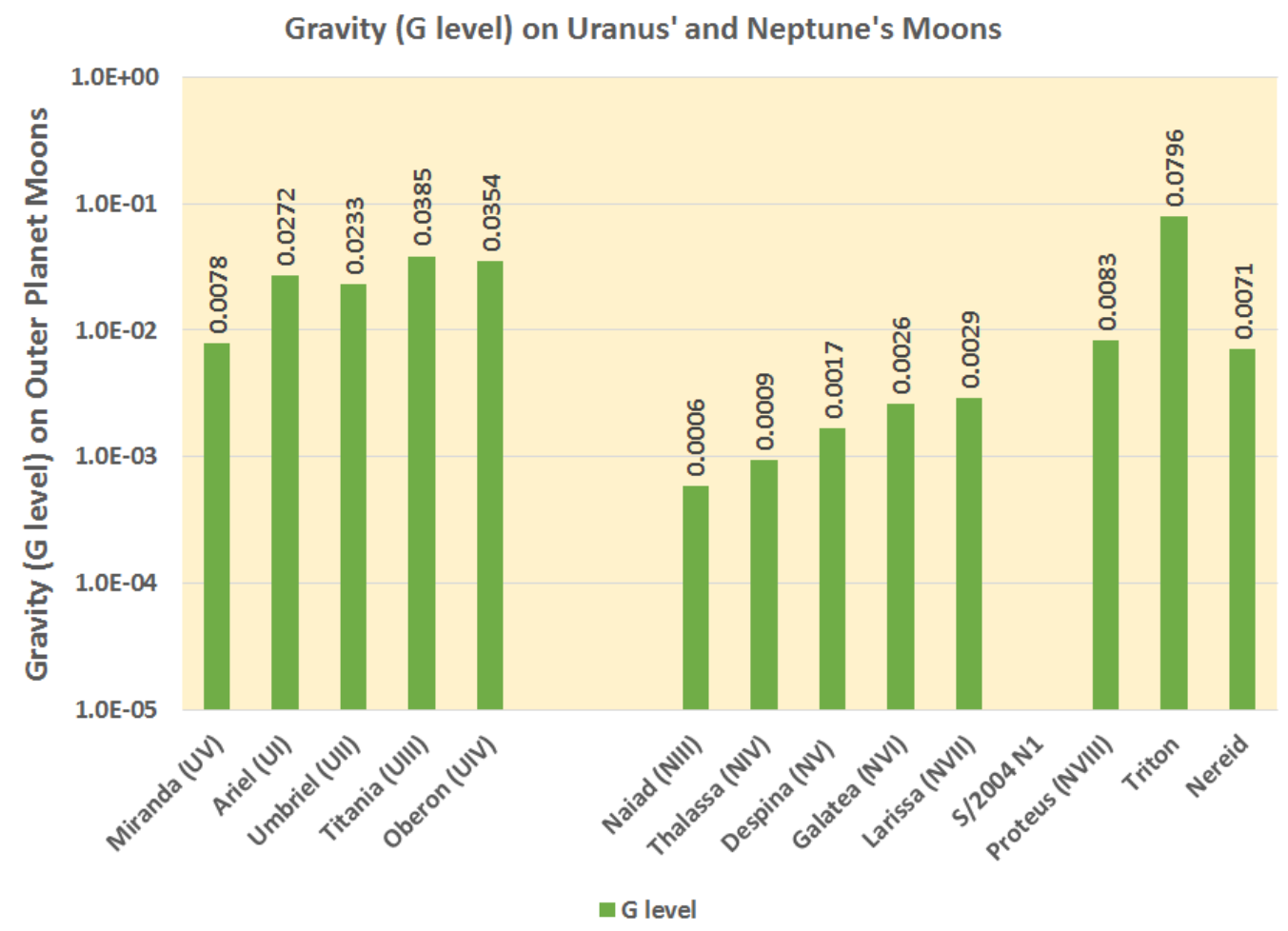

Figure 1. G on the Moons of Uranus and Neptune (based on Earth gravity) Levels 
For an in-space base, materials would have to be lifted into orbit to conduct the propellant processing. Alternatively, the propellant processing of water ice could be conducted on a moon's surface, thereby not wasting propellant resources by sending raw materials into orbit. Using an orbiting facility for the PPack factory may be more attractive. Landing the nuclear materials on the moon and then launching the finished product into space may be wasteful of precious propellant resources.

Artificial gravity space base assessments and ISRU analyses are needed to determine the appropriate level for gravity for the needed processes. While separation of rock and water ice is likely best done in a Moon's gravity field, conducting nuclear processing and PPack production may or may not require 10-3 G gravity levels. It may be argued that the low $10^{\wedge}-3 \mathrm{G}$ levels may make many processes difficult, and thus centrifuges (or other simulated gravity device) may be needed in orbit or on the moons to separate the rock and water ice on a moon's surface. In orbit, the PPack processes may require centrifuges, but on a smaller scale.

\section{F. OTV Propulsion assumptions and sizing}

An OTV propulsion and power model was developed using historical data (Refs. 8 and 30 to 32). The Isp and efficiency of the electric propulsion systems were 5,000 seconds with thruster efficiencies of $50 \%$ for each design. These design points are typical of advanced designs of either magneto-plasma-dynamic (MPD) or pulse inductive thrusters (PIT). While hydrogen is suggested for both thrusters, the possibilities of the higher Isp option using inert gases (xenon, krypton, etc.) is also viable. While hydrogen is more plentiful on the icy moons, inert gases have been detected on the Earth's Moon; it is suggested that these gases may also be available at the outer planet moons.

As the delta- $\mathrm{V}$ is so large for the transportation system, chemical propulsion is impractical. The OTV sizing was conducted for a wide range of power levels: $0.5 \mathrm{MWe}$ to $30 \mathrm{MWe}$. Three nuclear reactor specific masses were used: 10, 20, and $40 \mathrm{~kg} / \mathrm{kWe}$. The thruster technology delivers an Isp of either 5,000 at an engine efficiency of 50 percent. These technology levels represent MPD thruster capabilities with hydrogen as the propellant. The OTV propulsion dry mass, apart from and in addition to the reactor mass, was 101 MT and the propellant tankage mass was $5 \%$ of the mass of the required propellant.

The dry mass scaling equation used was (Ref. 1):

Mdry,stage $(\mathrm{kg})=$ reactor specific mass $(\mathrm{kg} / \mathrm{kW}) \cdot \mathrm{P}(\mathrm{kWe})+0.05 \cdot \mathrm{Mp}(\mathrm{kg})+$ fixed mass $(\mathrm{kg})$

The low thrust OTV delta-V values are presented in Table IV (for Uranus) and Table V (for Neptune) for the round trip missions. This delta- $\mathrm{V}$ value can accommodate round trip flights from a moon to Uranus or from a moon to Neptune, respectively.

\section{G. OTV results:}

The initial masses of the OTVs were estimated and the results are provided in Appendix A-1. The 5,000 second Isp cases were would likely be selected if the goal were the fastest trip time (Ref. 1). For the Miranda mission, with the $10 \mathrm{MWe}$ power level, a $101 \mathrm{MT}$ dry mass, and the lightest OTV reactor $(10 \mathrm{~kg} / \mathrm{kWe})$, the trip time is 228 days. The longest trip time (with the heaviest reactor, $40 \mathrm{~kg} / \mathrm{kWe}$ ) for the $101 \mathrm{MT}$ dry mass and $10 \mathrm{MWe}$ case is 569 days. At Titania, with the $101 \mathrm{MT}$ dry mass, at a $10 \mathrm{MWe}$ power level with the lightest reactor, the shortest trip time is 334 days and with the heaviest reactor, the longest time is 832 days. At Thalassa, with the 101 
MT dry mass, at $10 \mathrm{MWe}$, the shortest trip time is 120 days (lightest reactor, $10 \mathrm{~kg} / \mathrm{kWe}$ ) and the longest trip time is 298 days (with the heaviest, $40 \mathrm{~kg} / \mathrm{kWe}$ reactor).

The power level of 10 MWe appears to be the most acceptable, with a 20 and 30 MWe power level being too high, with no significant trip time benefits over $10 \mathrm{MWe}$. The $0.5 \mathrm{MWe}$ cases have very long trip times, and were not attractive for most cases. Additional detailed results regarding OTV initial mass, propellant mass, and the (round trip) trip time required for OTV refueling are presented in Appendix A-1.

\section{H. Lander propulsion assumptions and sizing}

The lander's mission is to deliver hydrogen to the OTV and return to the moon with the helium 3 or deuterium payload(s). The round trip delta-V values are provided in Tables II and III, for Uranus and Neptune, respectively This $1.9 \mathrm{~km} / \mathrm{s}$ delta- $\mathrm{V}$ value represents the energy to reach escape conditions from the moon Titania and return to the moon. Thus, the lander has the capability to reach escape conditions to rendezvous with the OTV. The lander was designed with an oxygen hydrogen main propulsion system. The lander Isp was varied from 400 to 480 seconds. The dry mass scaling equation was (Ref. 1):

$$
\text { Mdry,stage }(\mathrm{kg})=\text { Mdry, coefficient } ・ \mathrm{Mp}(\mathrm{kg})
$$

where:

$$
\begin{aligned}
& \text { Mdry,stage = the stage dry mass, including residual propellant }(\mathrm{kg}) \\
& \text { Mdry,coefficient = the B mass coefficient ( } \mathrm{kg} \text { of tank mass / } \mathrm{kg} \text { of usable propellant mass) } \\
& \text { Mp = Propellant mass }(\mathrm{kg})
\end{aligned}
$$

where $\mathrm{B}=0.4 \mathrm{Mp}$.

The value of the B parameter would be typical of landers with smaller propellant loads. The payload masses for the analyses were 50,100, and 200 MT. The tankage to contain the payload of fuel is 10 percent of that payload mass. The landers are sized to carry the full payload of fuel on a round trip flight. This is the worst case for abort scenarios, where the lander may miss an OTV refueling rendezvous and have to return its full payload to the moon.

\section{Lander results}

The lander initial mass, propellant mass, and number of flights are shown in the Appendix A-2 and in Appendix A-1, the number of flights required is presented. In some cases, the lander propellant masses are actually quite modest. The following results are for the Miranda lander, with a B coefficient of 0.4. For the $50 \mathrm{MT}$ payloads with a 400-s Isp, the propellant masses are 7.9 MT and with 200 MT of payload, the propellant mass is 32 MT. With a 480-s Isp, the propellant masses are 6.5 MT and 26 MT, respectively. 
For Titania, with a B coefficient of 0.4, and a 50 MT payload and an Isp of $400 \mathrm{~s}$, the propellant mass is 45.6 MT; for the 200 MT payload, the propellant mass is 182.5 MT. With a B coefficient of 0.4 , and a 50 MT payload and an Isp of $480 \mathrm{~s}$, the propellant mass is $34.1 \mathrm{MT}$; for the same coefficient and Isp, with the $200 \mathrm{MT}$ payload, the propellant mass is 136.5 MT. Many additional results regarding lander mass, propellant mass, and number of flights required for OTV refueling are presented in Appendix A-2 and A-1.

Certainly, the 200 MT payload lander flights require the fewest flights. Transferring all of the OTV propellant in one refueling will seemingly be more effective than awaiting 2 or 4 times the number of lander refueling flights. Power aboard the OTV can help maintain the cryogenic liquids at their appropriate temperatures and pressures during the propellant transfers.

\section{J. In-space (space base) factory masses}

Due to the extremely low gravity on the outer planet moons, in-space processing with artificial gravity was assessed. Several historical space base designed were reviewed and their configurations were modified to accommodate large scale propellant processing (Refs. 37 and 38). The space bases would be placed in orbit about the outer planet moon. Deliveries of raw materials would be made from the outer planet moon's surface.

The space base masses that contain the factories can be in several different configurations. The range of masses, from the minimal robotic option to the highest masses for human tended bases, are shown in Figure 2.

Appendix $\mathrm{C}$ provides the mass summaries for each option. For the outer planets, the smaller robotic options may be the most attractive. With smaller robotic bases, the overall transportation costs will be reduced. The power levels of the robotic space bases were between 1 and $5 \mathrm{MWe}$. The mass of $50 \mathrm{MT}$ was allocated to the power system, and the resultant power level is based on the technology levels of 10,20 , and $40 \mathrm{~kg} / \mathrm{kWe}$. If the technology level is $40 \mathrm{kw} / \mathrm{kWe}$, the total power level available is $1.25 \mathrm{MWe}$. If the available technology level is $10 \mathrm{~kg} / \mathrm{kWe}$, the power level would be $5 \mathrm{MWe}$.

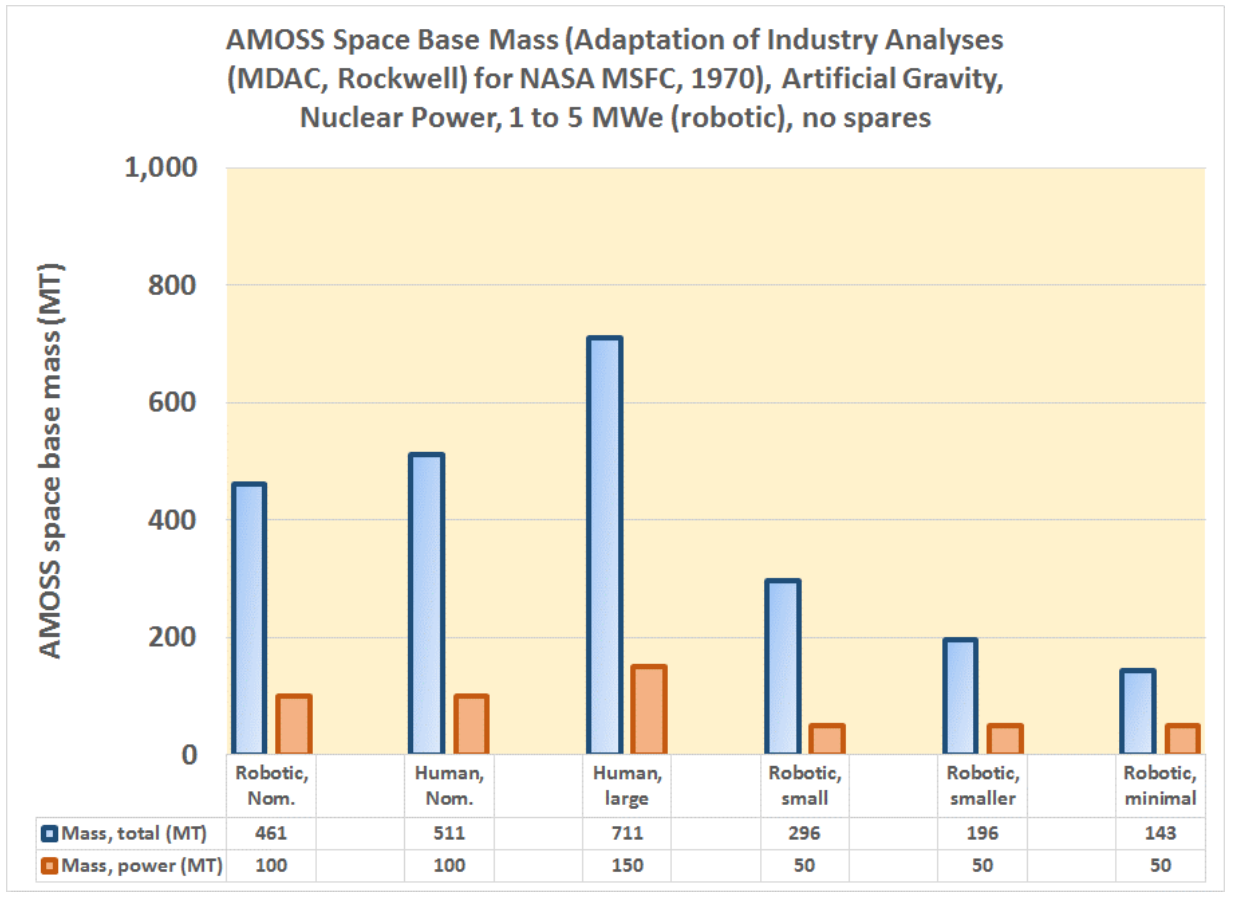

Figure 2. Space base mass estimates (based on extrapolations of data in Ref. 37 and 38) 


\section{Preliminary Optimization(s) and Observations}

Establishing an optimum transportation system will be influenced by many factors: the OTV mass and power level, the payload mass of the lander and the selection of the moon for the mining factories. Several optima will be created based on the size and mass of the moon selected. The moon's mass will strongly influence the propellant mass needed for the refueling of its oxygen/hydrogen propulsion system and the time needed for creating the fuel for the OTV.

Four examples of the estimated transportation system masses are presented in Figures 3 and 4 for Uranus and Figures 5 and 6 for Neptune. The OTV and lander masses for all of the major moons of each planet were assessed. As noted, these moons represent the moons with the smallest and largest delta- $\mathrm{V}$ values required for moon landing operations at each planet. Figures 3 and 4 represent the cases with the NEP OTV operating with an Isp of $5,000 \mathrm{~s}$ and a reactor alpha of 10 and $40 \mathrm{~kg} / \mathrm{kW}$, respectively. Figure 3 shows the transportation system mass for a lighter reactor, with an alpha of $10 \mathrm{~kg} / \mathrm{kW}$. For all cases, a lander Isp value of $460 \mathrm{~s}$ was selected for the comparison. Many other such comparisons can be created from the large set of data presented in Appendices A-1 and A2. .

In comparing the Uranus cases in Figures 3 and 4, the strong influence of the NEP OTV reactor mass is clear. By using the lighter reactor, the overall transportation mass will be reduced. A $40 \mathrm{~kg} / \mathrm{kW}$ OTV for Miranda has a mass of $716 \mathrm{MT}$ and by using a $10 \mathrm{~kg} / \mathrm{kW}$ reactor, this OTV mass can be reduced to $287 \mathrm{MT}$. Similar trends are noted in the Neptune cases in Figures 5 and 6. Generally, the smaller moon closer to the planet are more mass efficient, requiring smaller OTV and smaller landers.

With the OTVs, the 10 MWe power level appears to be the most acceptable. The initial mass of the OTV with power levels of 20 and $30 \mathrm{MWe}$ is too high in comparison with the $10 \mathrm{MW}$ cases, with no significant trip time benefits over the OTV at the $10 \mathrm{MWe}$ power level. For the $101 \mathrm{MT}$ dry mass case for Miranda, at $10 \mathrm{~kg} / \mathrm{kWe}$, and at 5,000 seconds of Isp value, the trip time for the $30 \mathrm{MW}$ level is 152 days versus 228 days at the $10 \mathrm{MW}$ level. With the $40 \mathrm{~kg} / \mathrm{kWe}$ case (with the same Isp and dry mass), the trip time at $30 \mathrm{MWe}$ is 493 days versus 569 days for the $10 \mathrm{MWe}$ case. The OTV trip times are a significant issue. Many flight times are 100's of days. Initially, a single 1 MT payload of helium 3 or deuterium would fly on each OTV flight. Multiple helium 3 or deuterium payloads will have to be manifested on the OTVs.

While the OTV and the lander can rendezvous at the moon's escape conditions, it may be more stable to conduct the propellant and payload transfers at a high moon orbit, but not at or beyond the moon's escape conditions. The OTV trip time to enter and establish a suitably stable orbit for the propellant transfer from the lander will increase, but that increase is likely a small number of days.

Lander payloads of 200 MT provide the minimal number of lander flights. Small lander payloads may be acceptable, but the processing on the moon of the propellant, the propellant loading, and the cryogenic hydrogen loading may favor the largest payload capacity lander. With the 200 MT hydrogen payload, the number of lander flights needed to refuel the $101 \mathrm{MT}$ dry mass (5,000 seconds Isp) OTV for Miranda is 1 flights for the $10 \mathrm{~kg} / \mathrm{kWe}$ case and 2 flights for the $40 \mathrm{~kg} / \mathrm{kWe}$ case. For the $50 \mathrm{MT}$ lander flights, the numbers of flights were 2 for the 10 $\mathrm{kg} / \mathrm{kW}$ case and 5 for the $40 \mathrm{~kg} / \mathrm{kW}$ case. Landers might be further optimized by increasing their payload capacity, which would further reduce the number of flights.

Fuel processing, and robotic storage and transportation of helium 3 and deuterium will likely be a major challenge. Physics Package (PPack) factory will have large uncertainties in its mass estimate. If the mass from Earth becomes too high (making the ISRU case potentially untenable or unsustainable), it is likely that the factory itself can be created from in-situ resources. Ice and rock from moon mining and water extraction and purification will be required. Selecting a small moon with minimal gravity may be another challenge, and experiments will be 
needed to prove which gravity level is sufficient for mining operations (i.e., is $0.01 \mathrm{G}$ too low a gravity level for this processing?).

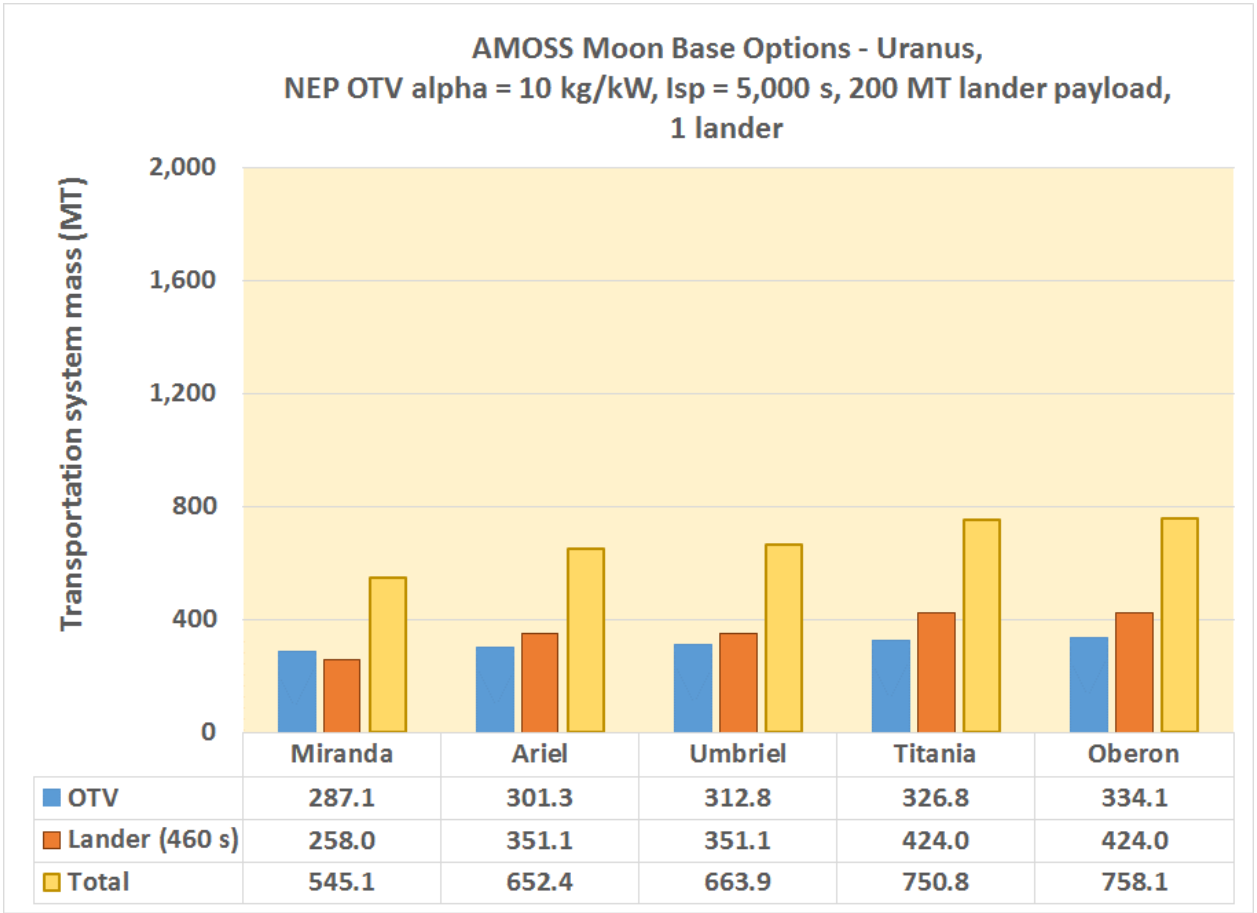

Figure 3. Uranus - Transportation System Mass - NEP alpha $=10 \mathrm{~kg} / \mathrm{kW}$, Isp $=5,000 \mathrm{~s}$, Lander Isp $=460 \mathrm{~s}$ (one lander included in each case).

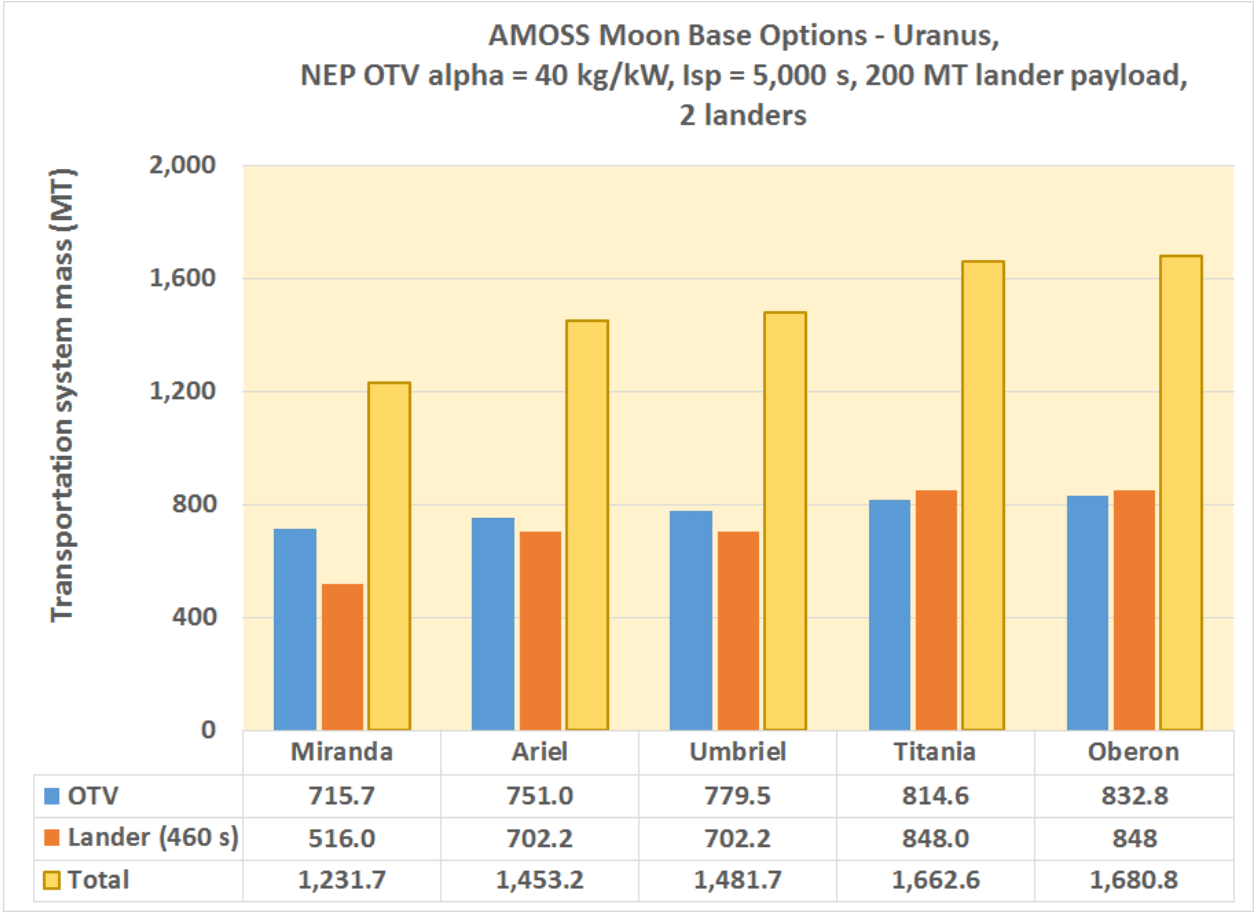

Figure 4. Uranus - Transportation System Mass - NEP alpha $=40 \mathrm{~kg} / \mathrm{kW}$, Isp $=5,000 \mathrm{~s}$, Lander Isp $=460 \mathrm{~s}$ (one lander included in each case). 


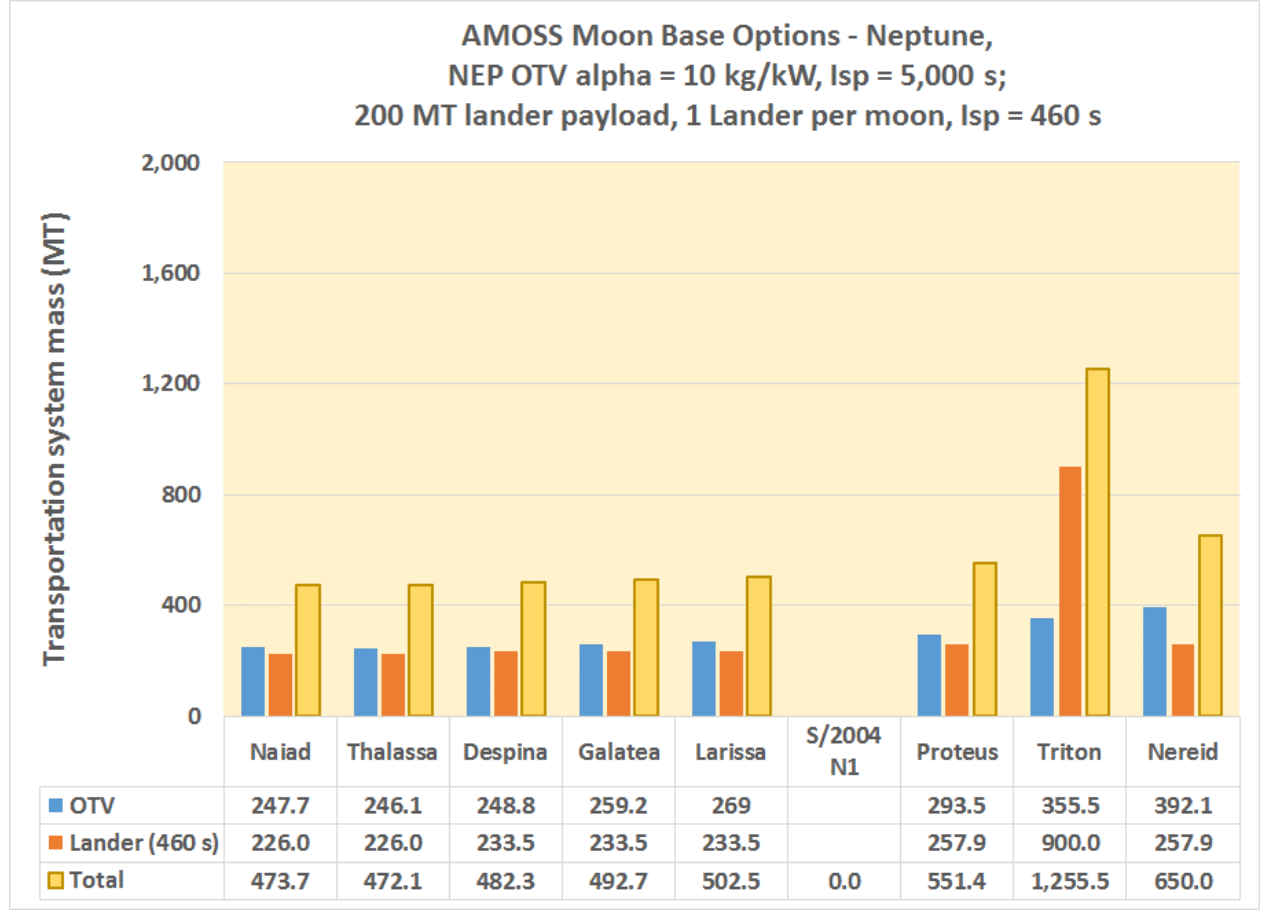

Figure 5. Neptune - Transportation System Mass - NEP alpha $=10 \mathrm{~kg} / \mathrm{kW}$, Isp $=5,000 \mathrm{~s}$, Lander Isp $=460 \mathrm{~s}$ (one lander included in each case).

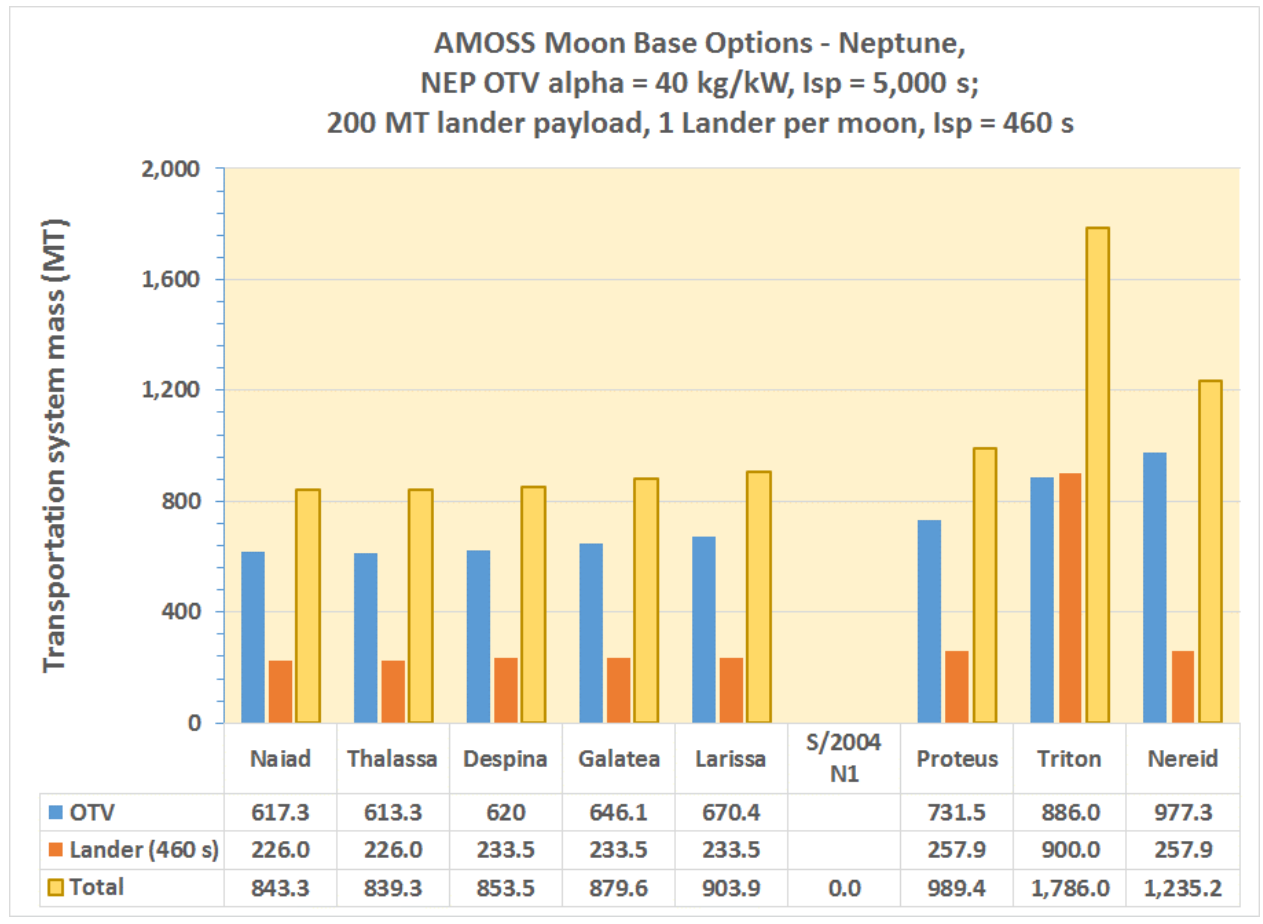

Figure 6. Neptune - Transportation System Mass - NEP alpha $=40 \mathrm{~kg} / \mathrm{kW}$, Isp $=5,000 \mathrm{~s}$, Lander Isp $=460 \mathrm{~s}$ (one lander required in all other cases). 
To see the influence of replacement scheduling, an overall architecture assessment was conducted. Based on the vehicle mass data from Refs. 1 to 12, a set of nominal masses were selected that are representative of the major architecture components. Those components are shown in Figure 7. In Figure 8, the total mass of the architecture over a 28 year period is shown. There are two major summations: I for initial deployment and R for replacement components. This mass summation is representative of the masses needed if the elements must be replaced every 7 years. The masses shown in the figures do not include the interplanetary transfer vehicles (ITV) needed for the flight to Uranus or Neptune. Over 11,000 (replacement masses) to 14,000 MT (initial masses) of vehicles would be needed over the 28 year period. The 7 year period for replacement was selected based on the lifetime of reactors noted for the SP-100 space nuclear reactor systems (Ref. 39).

In Figures 9 and 10, the total number of OTV flights that are possible for a 7 and 10 year OTV operating life are depicted. It is clear that the smaller moons near the planet, requiring shorter flight times will be able to accomplish more round trip flights in the 7 to 10 year reactor lifetimes. In Figure 9, the Miranda OTV is able to conduct 11 round trip in the 7 year lifetime. For Titania, only 7 round trips are possible in the 7 year reactor lifetime. Similarly, in Figure 10, the Thalassa OTV can complete 21 round trips while the Triton OTV can only complete 6 round trip flights in the 7 -year reactor lifetime.

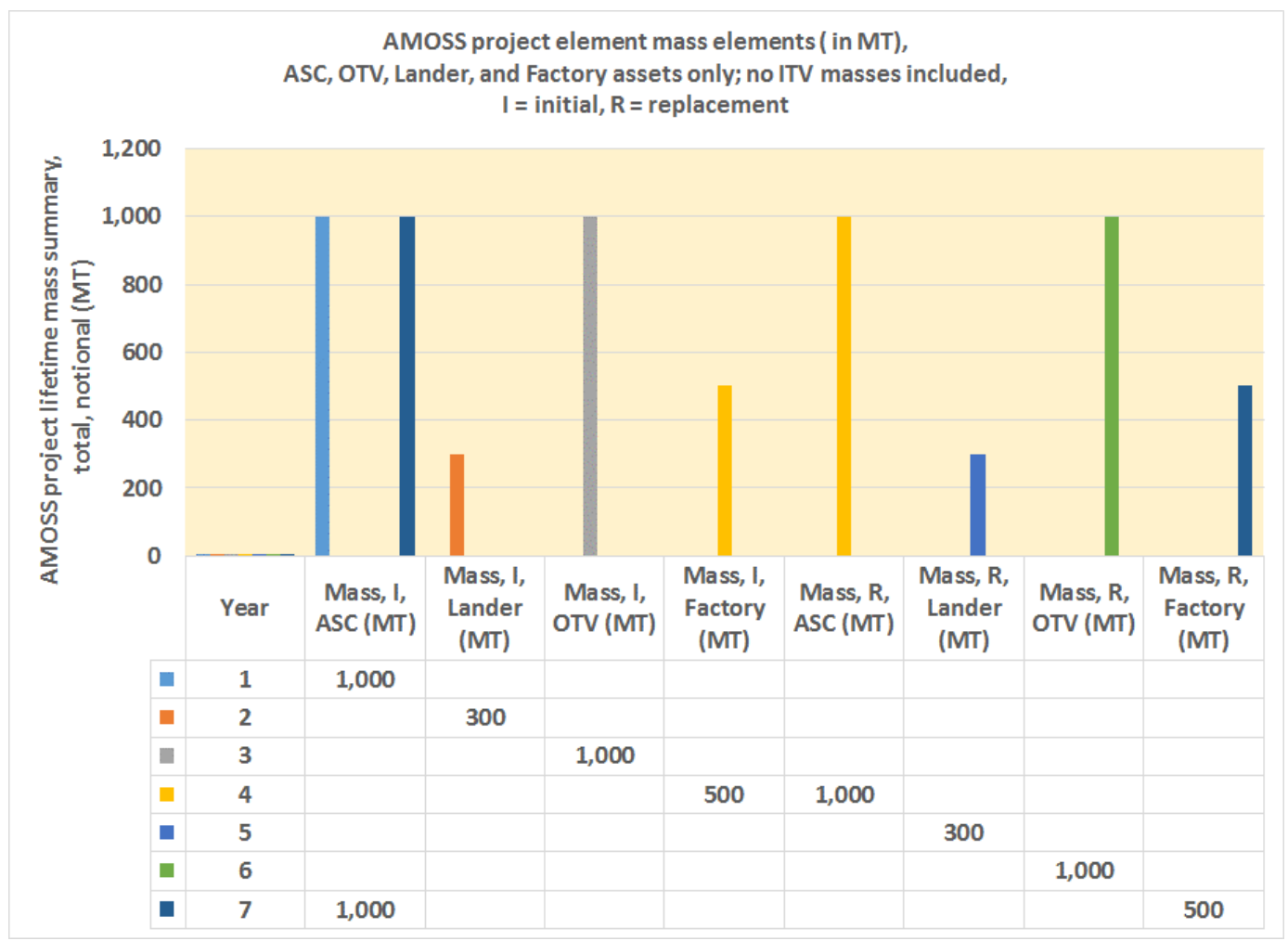

Figure 7. AMOSS on-orbit mass requirements for individual elements, nominal case. 


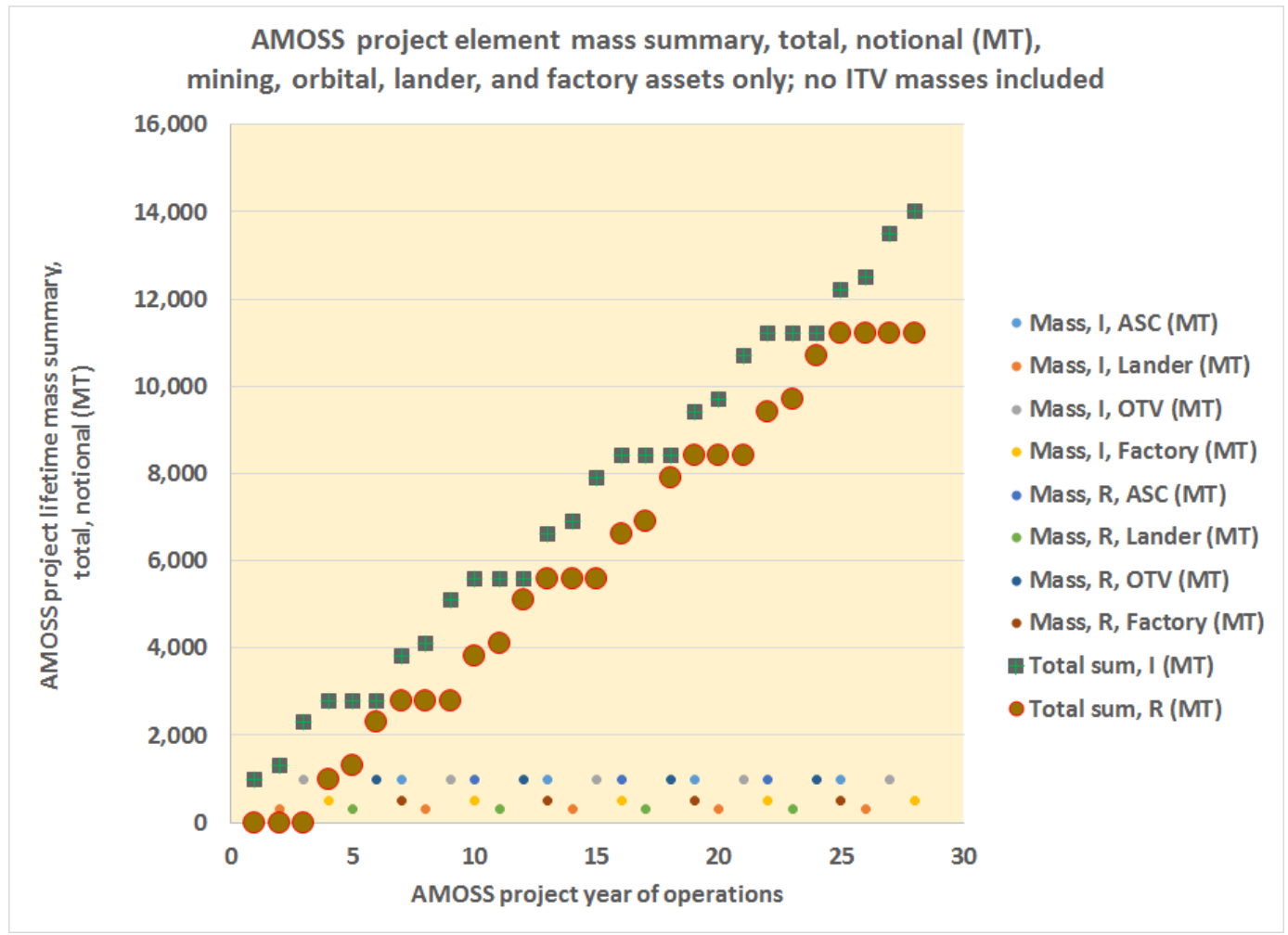

Figure 8. AMOSS on-orbit mass requirements for 28 years of operations, nominal case.

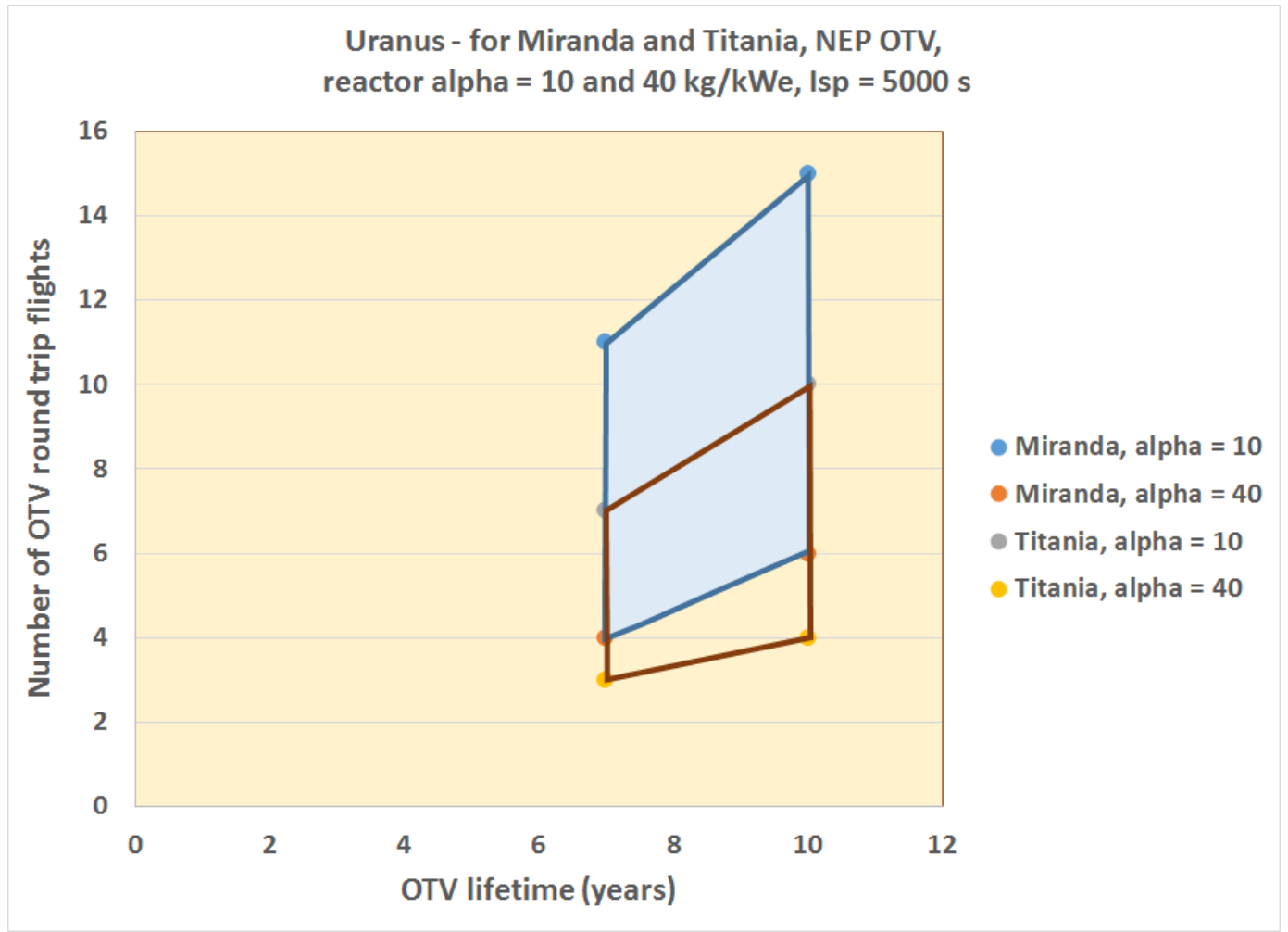

Figure 9. At Uranus - Number of round trip OTV flights for Miranda and Titania. 


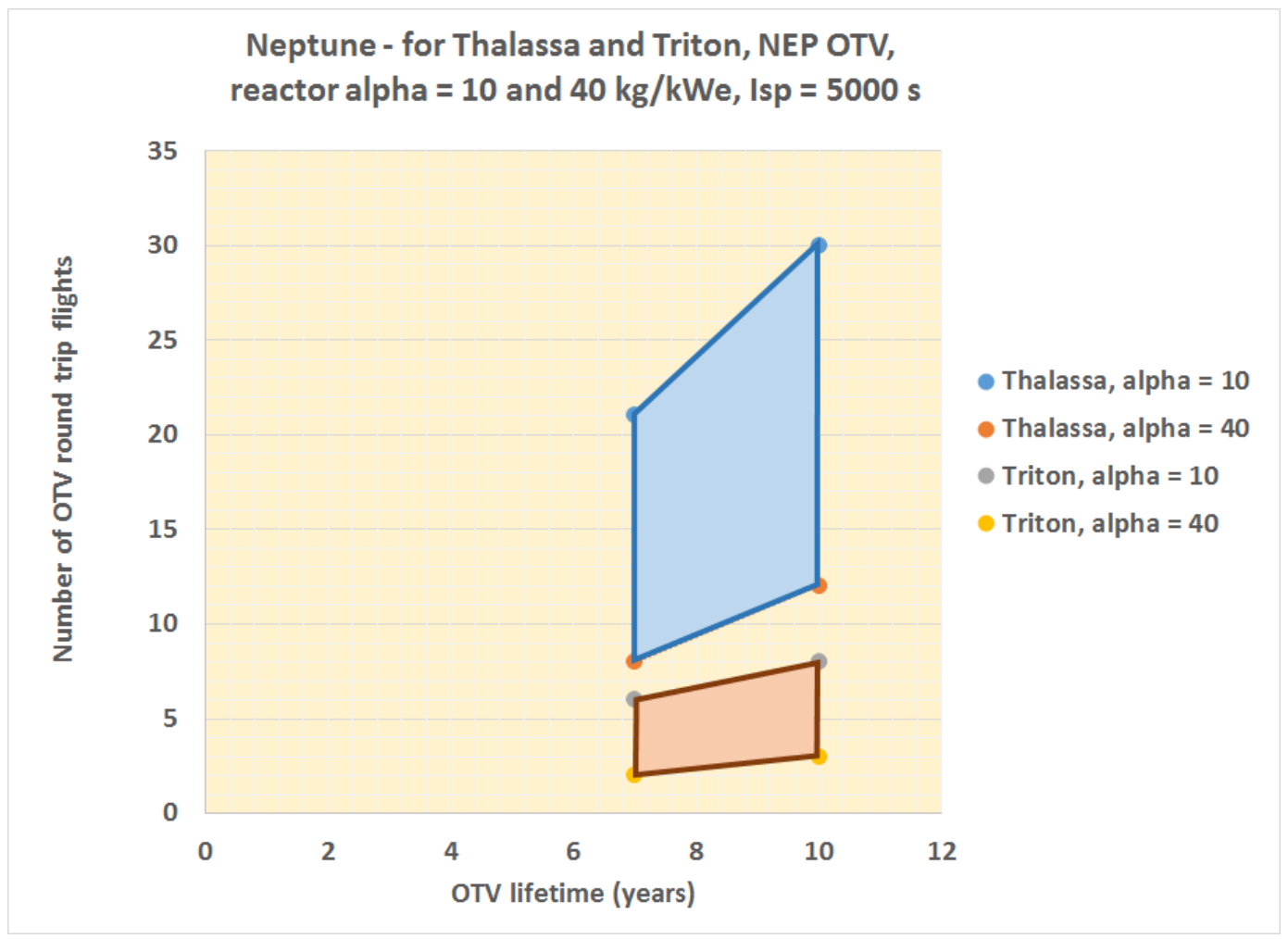

Figure 10. At Neptune - Number of round trip OTV flights for Thalassa and Triton

\section{Concluding Remarks}

Atmospheric mining in the outer solar system has many significant benefits. The use of ISRU in the outer solar system will entail wresting nuclear fuels from Uranus and or Neptune. Using outer planet moon bases for mining the propellants for OTVs and landers is an important option. Storing the AMOSS nuclear fuels away from the atmosphere will minimize the potential for unanticipated deorbiting of the orbiting storage facility. Using the moons for storage of the nuclear fuels and base of operations for OTV refueling is an excellent option. Though the gravity of these moons are much lower than that of Earth, that gravity will assist in any processes for mining and fuel processing. The $10 \mathrm{MWe}$ power levels for the OTV seems best for providing a relatively short moon-to-planet trip time. The OTVs and landers will rendezvous near the escape condition of the small moon, shortening the trip time for the OTV (eliminating the need to spiral into low moon orbit). Larger landers (of 200 MT payloads) are more attractive than small landers, as the large landers require fewer flights to resupply the OTVs with fuel. The OTV trip times may be too long for effective use of the more distant outer planet moons. Moons that are closer to the planet may be required. Generally, the smaller moon closer to the planet are more mass efficient, requiring smaller OTV and smaller landers.

Both Uranus and Neptune transportation system mass estimates are presented in Figures 3 and 4 for Uranus, and Figures 5 and 6 for Neptune. In each figure, the major moons are noted and the OTV and lander masses are shown. Of the 5 major moons of Uranus, the moons closest to the planet required the lowest transportation system mass. For Uranus, Miranda has the lowest transportation system mass. The lander mass has a strong influence on the overall mass. The result is similar for the 8 major moons of Neptune. The transportation system for Thalassa has the lowest overall mass of the 8 Neptune moon destinations. Thalassa is the moon with the lowest gravity, allowing the lander to be much smaller than any of the 4 cases. Therefore, increasing the lander Isp or using 
a moon with reduced gravity has a powerful effect on the overall mass. Reducing the NEP OTV reactor mass also has a strong influence on reducing the overall transportation system mass.

The transportation masses for both the largest and the smallest moons of Uranus and Neptune were investigated. At both Uranus and Neptune, the smaller moons near the planet had the lowest transportation mass. While this is an important result, it is also critically important to address the need for additional gravity for processing of fuels and propellants. Centrifuges (or other equally effective technologies) to separate the water ice and rocky feed stocks from the outer planet moon's surface will likely be needed.

The overall architecture masses were estimated for a range of replacement schedules. The use of artificial gravity space bases or centrifuges on the moon bases will very likely be required. The low gravity of the outer planet moons will not be sufficient to process the ISRU related materials and allow efficient production of OTV and lander propellants. The masses of the robotic in-space bases with artificial gravity were in the range of 143 to 461 MT. These added space base masses does not significantly increase the overall architecture masses, and they truly make the architecture more viable than one without artificial gravity.

Further optimizations and creative usage of propulsion technologies will likely lead to great improvements in the transportation systems and reduce its cost and mass. The exploration of the outer planets and their ultimate use in powering future fusion powered space vehicles will allow unlimited options for solar system exploration, and ultimately, flights to the stars. 
Appendix A-1: NEP OTV and Moon Lander Data - Mass, Trip Time, Number of Lander Cargo Flights 101 MT payload and propulsion dry mass cases, 5,000 second Isp cases -

Figure A-1-1: Initial mass - Miranda OTVs

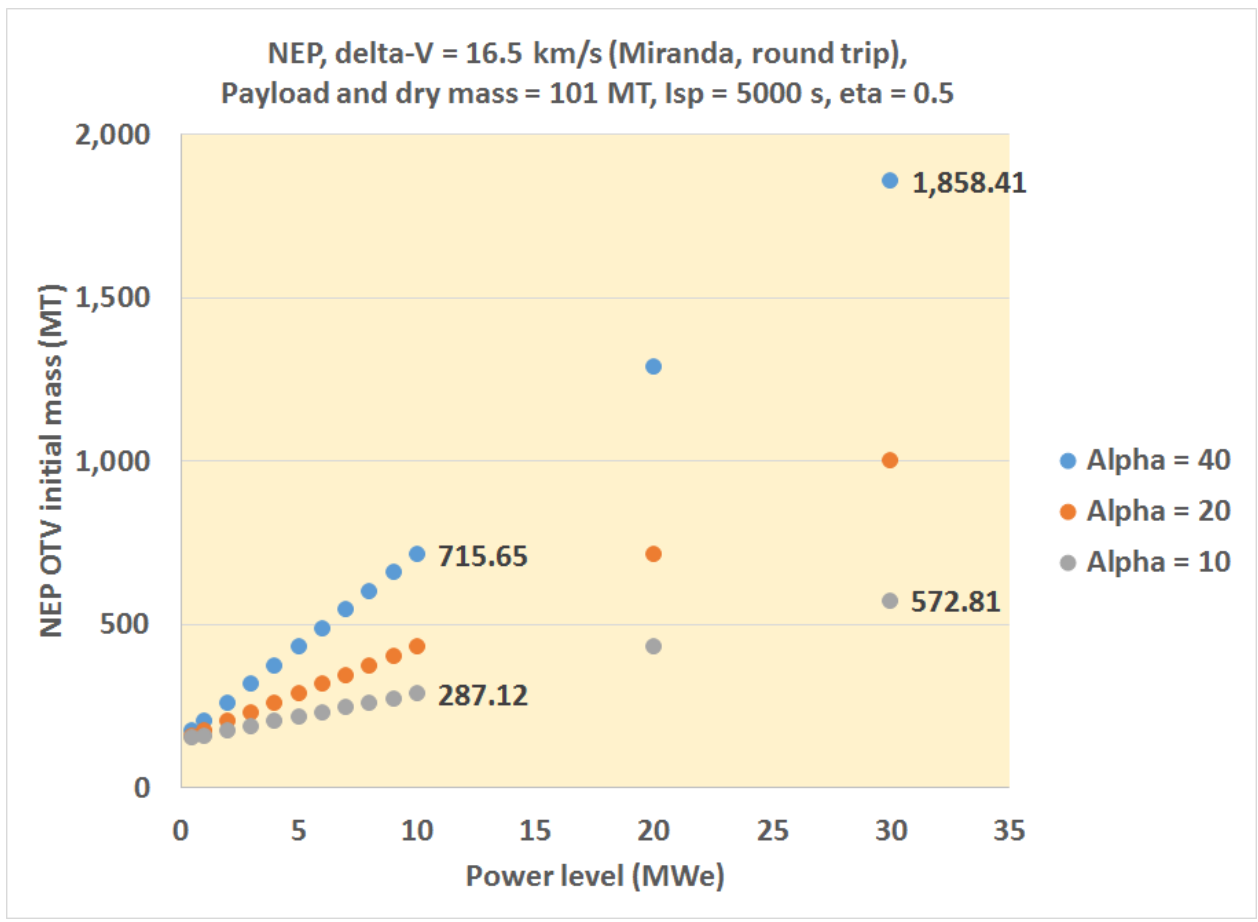

Figure A-1-2: Propellant mass - Miranda OTVs

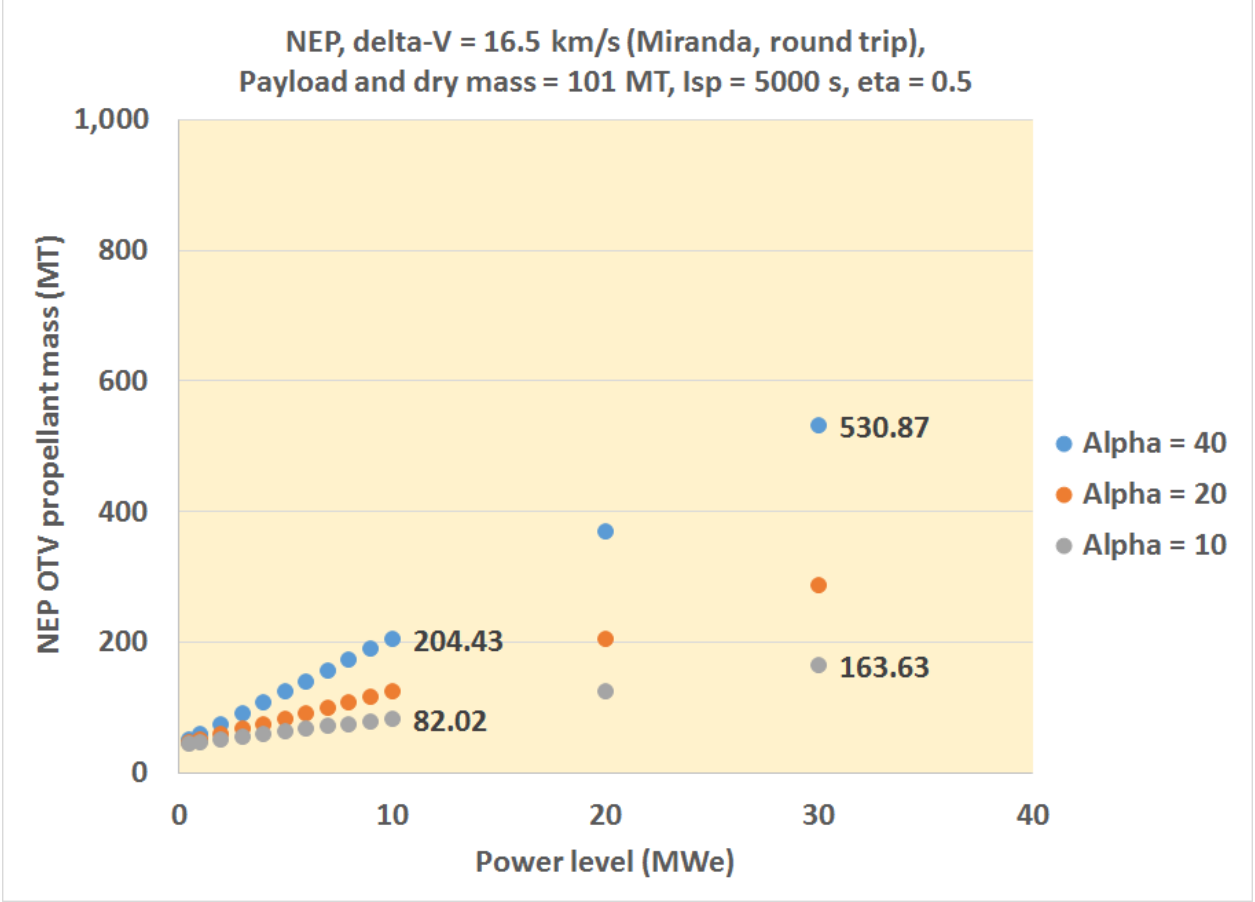


Figure A-1-3: Trip time - Miranda OTVs

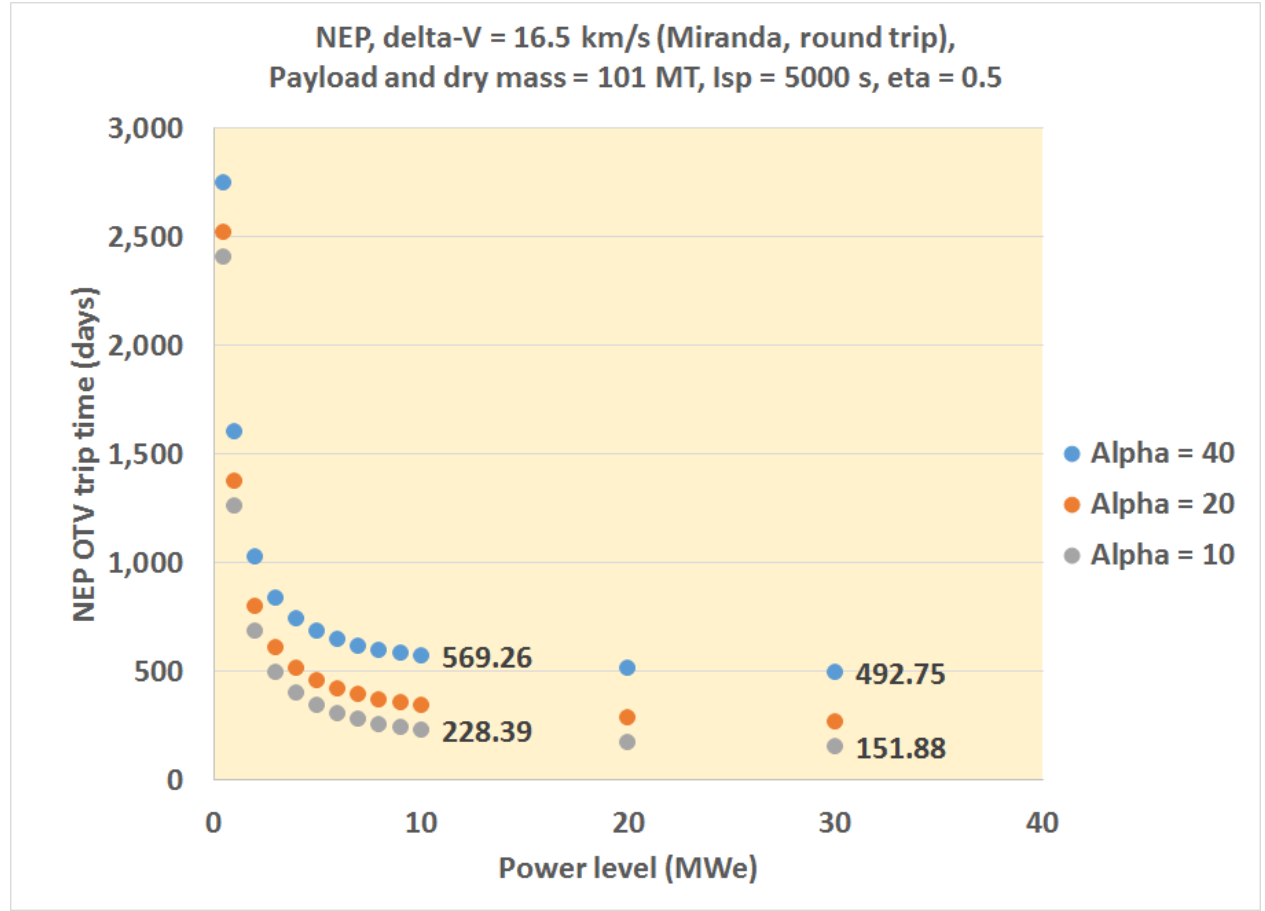

Figure A-1-4: Number of flights, 50 MT payloads - Miranda OTVs

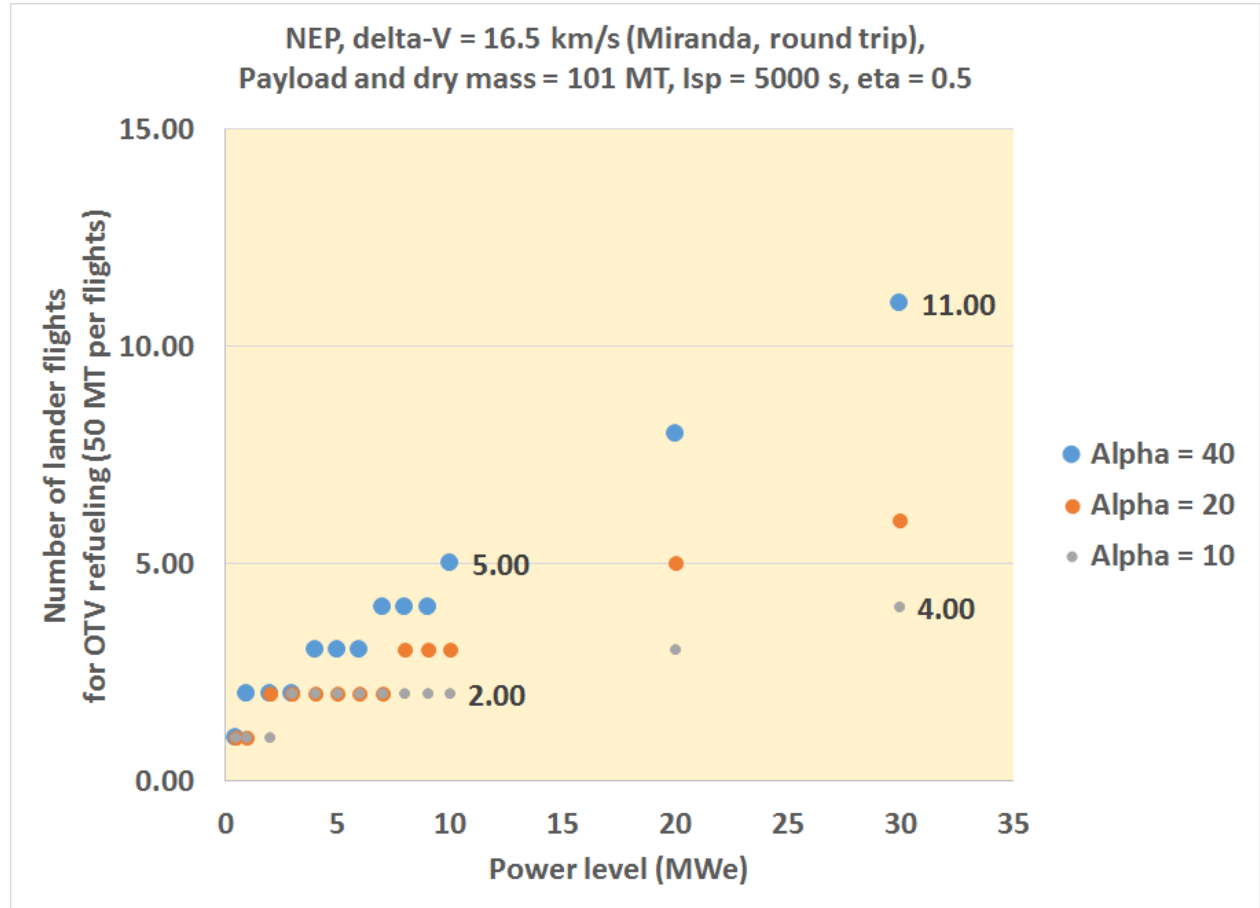


Figure A-1-5: Number of flights, 100 MT payloads - Miranda OTVs

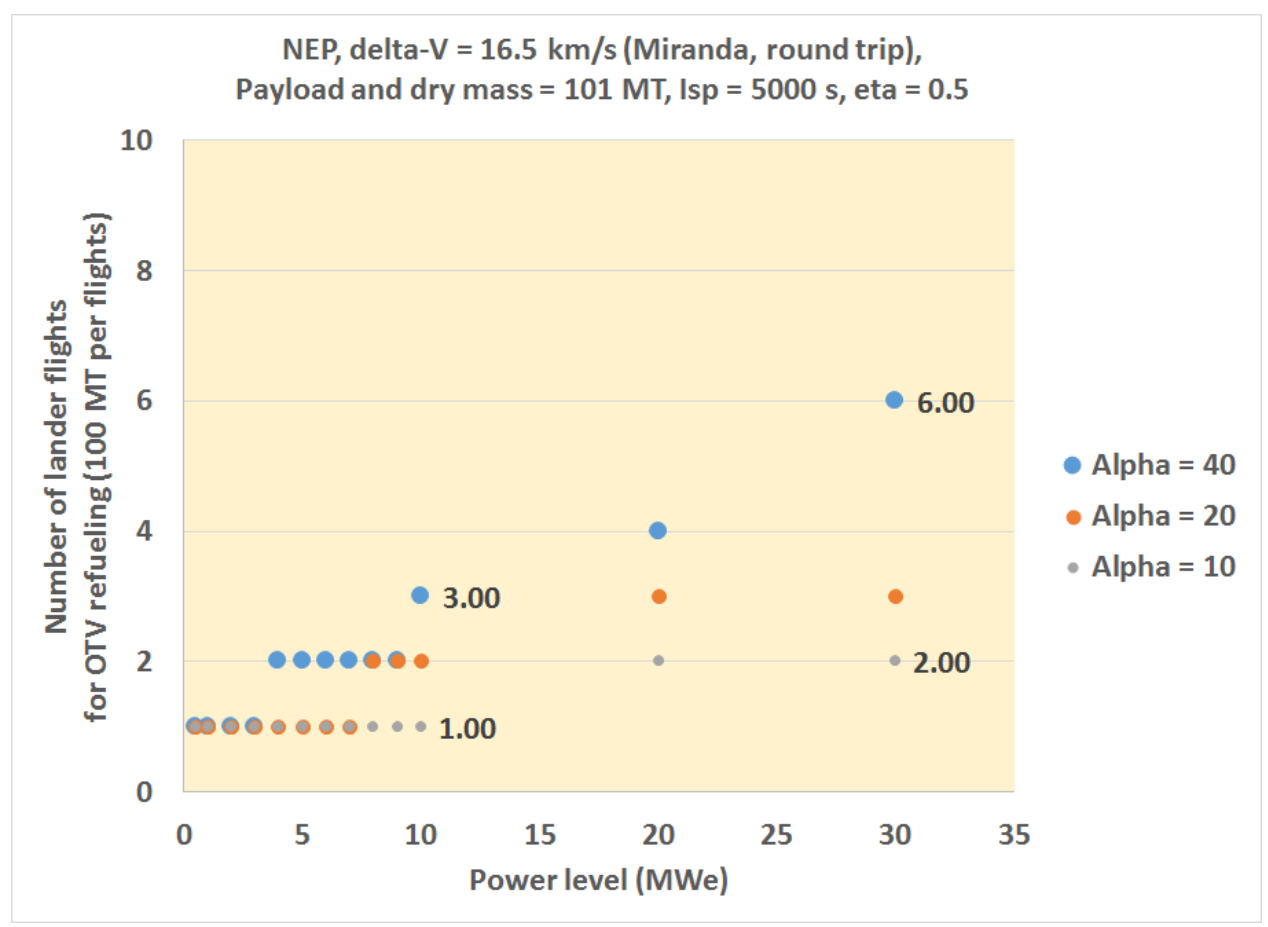

Figure A-1-6: Number of flights, 200 MT payloads - Miranda OTVs

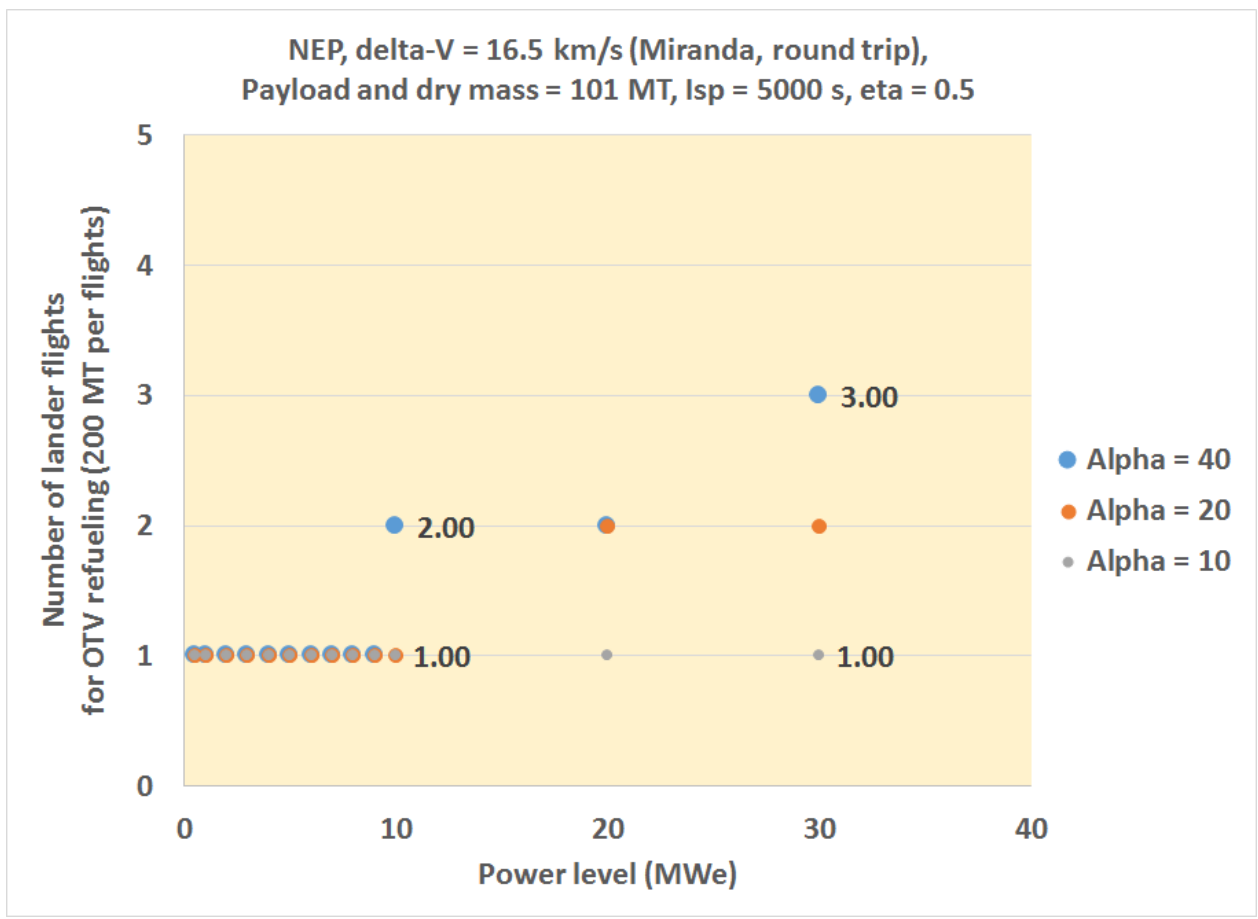


Figure A-1-7: Initial mass - Titania OTVs

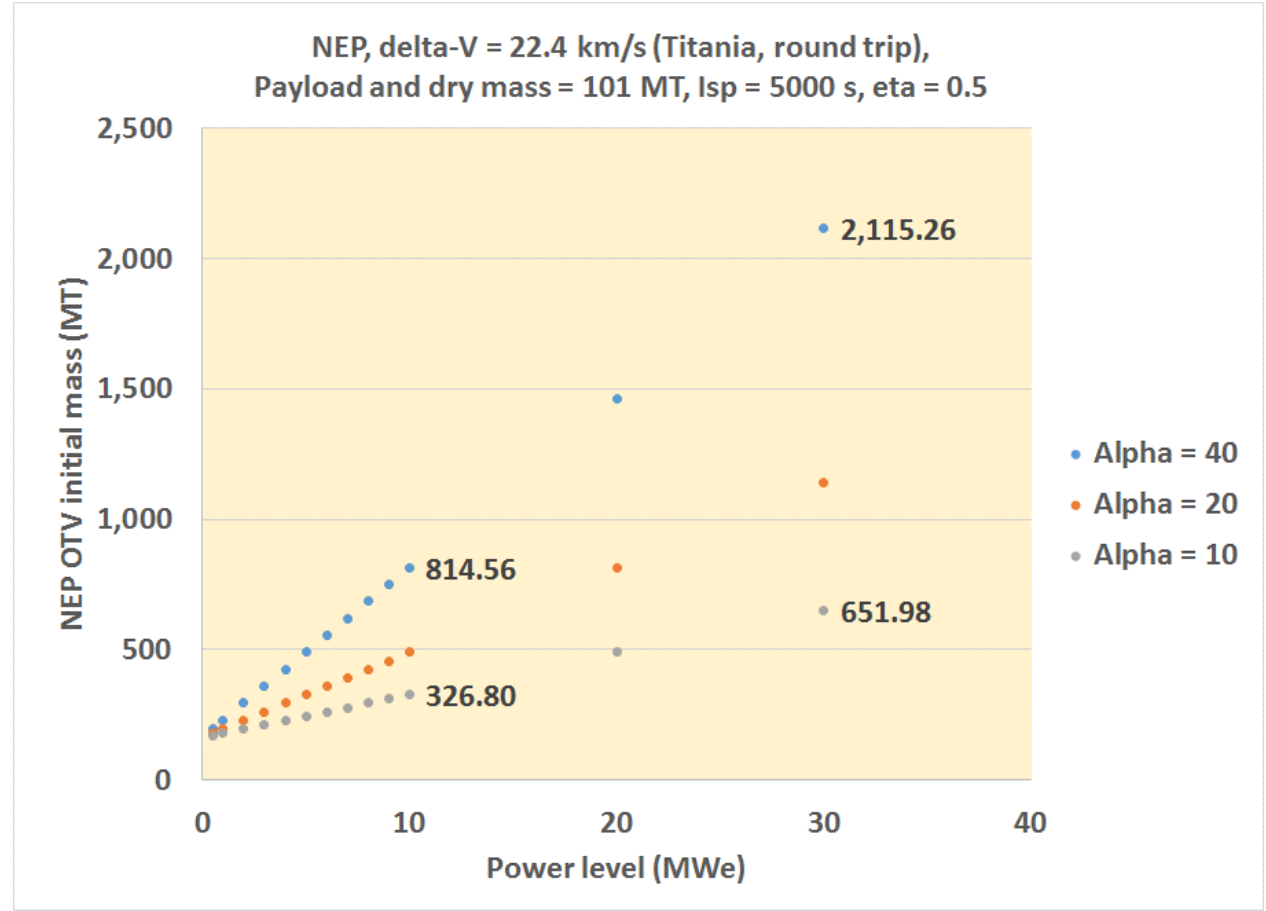

Figure A-1-8: Propellant mass - Titania OTVs

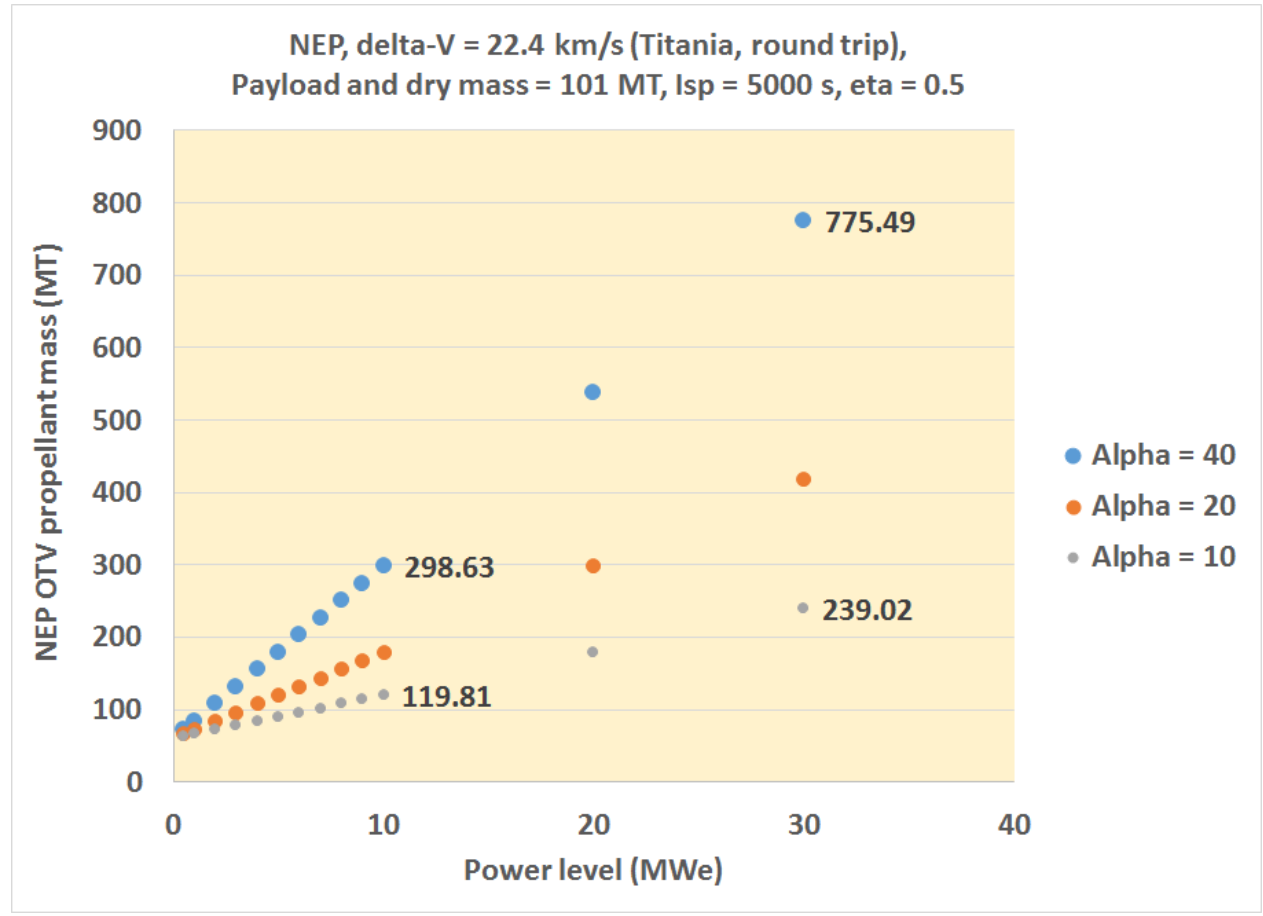


Figure A-1-9: Trip time - Titania OTVs

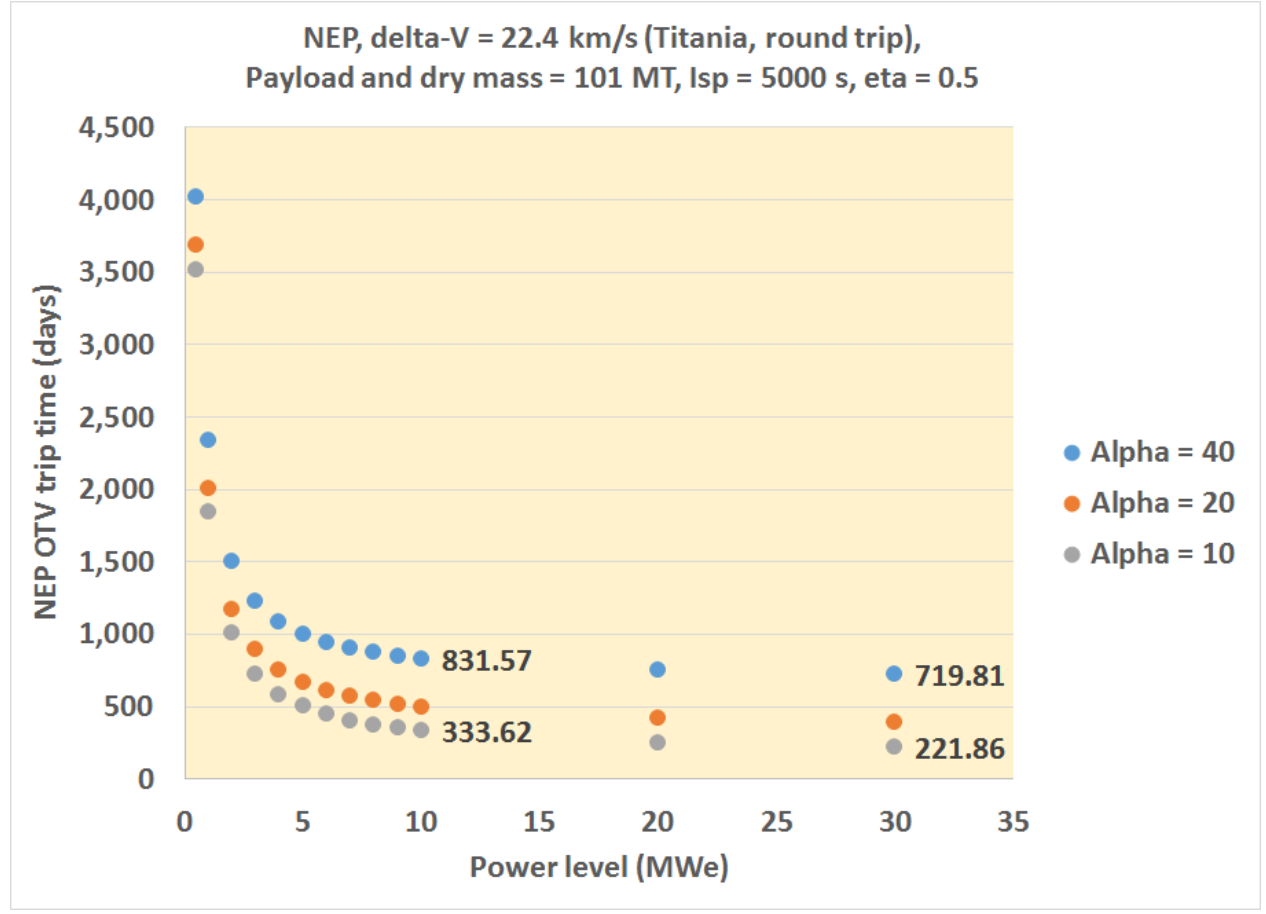

Figure A-1-10: Number of flights, 50 MT payloads - Titania OTVs

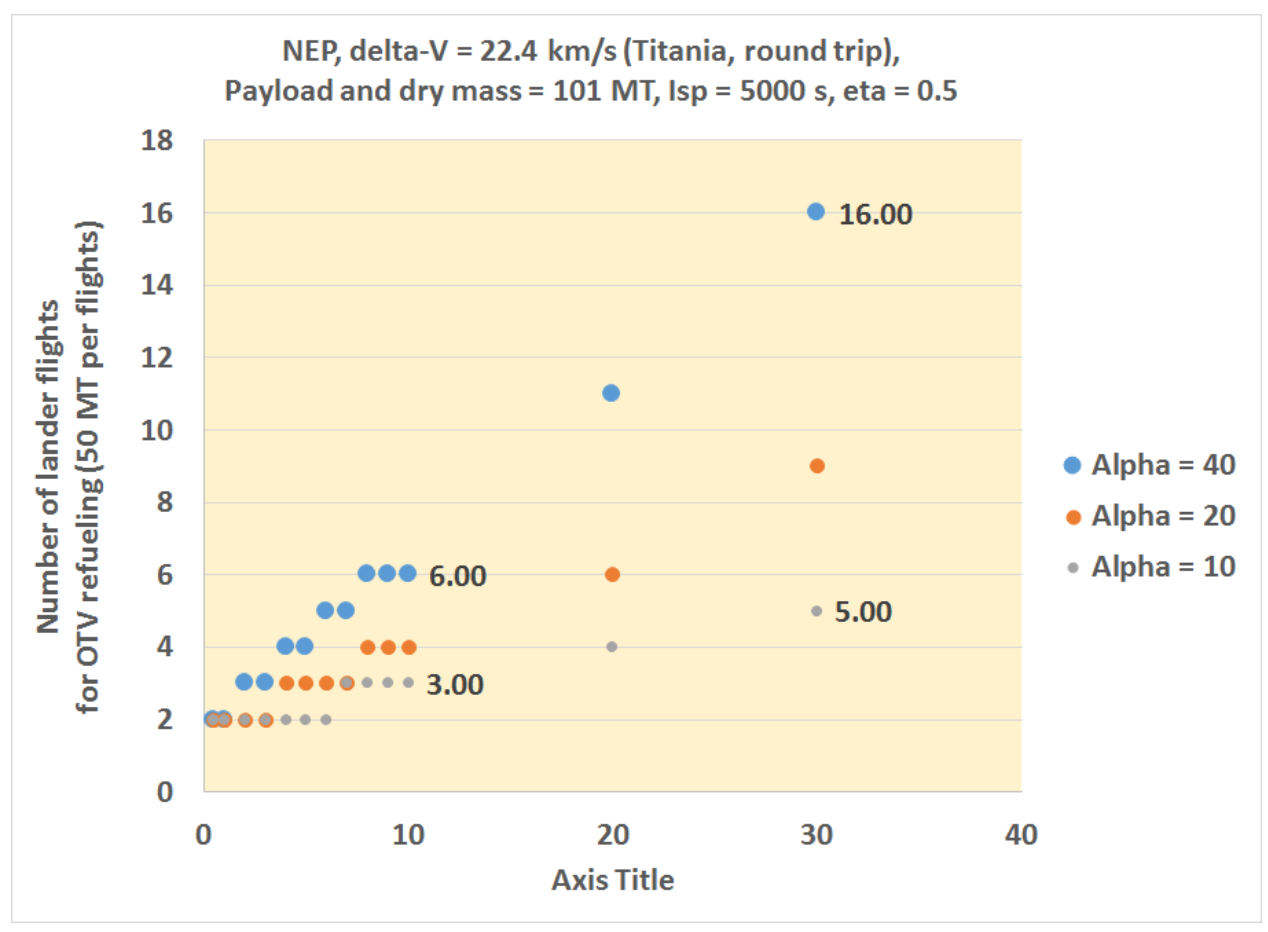


Figure A-1-11: Number of flights, 100 MT payloads - Titania OTVs

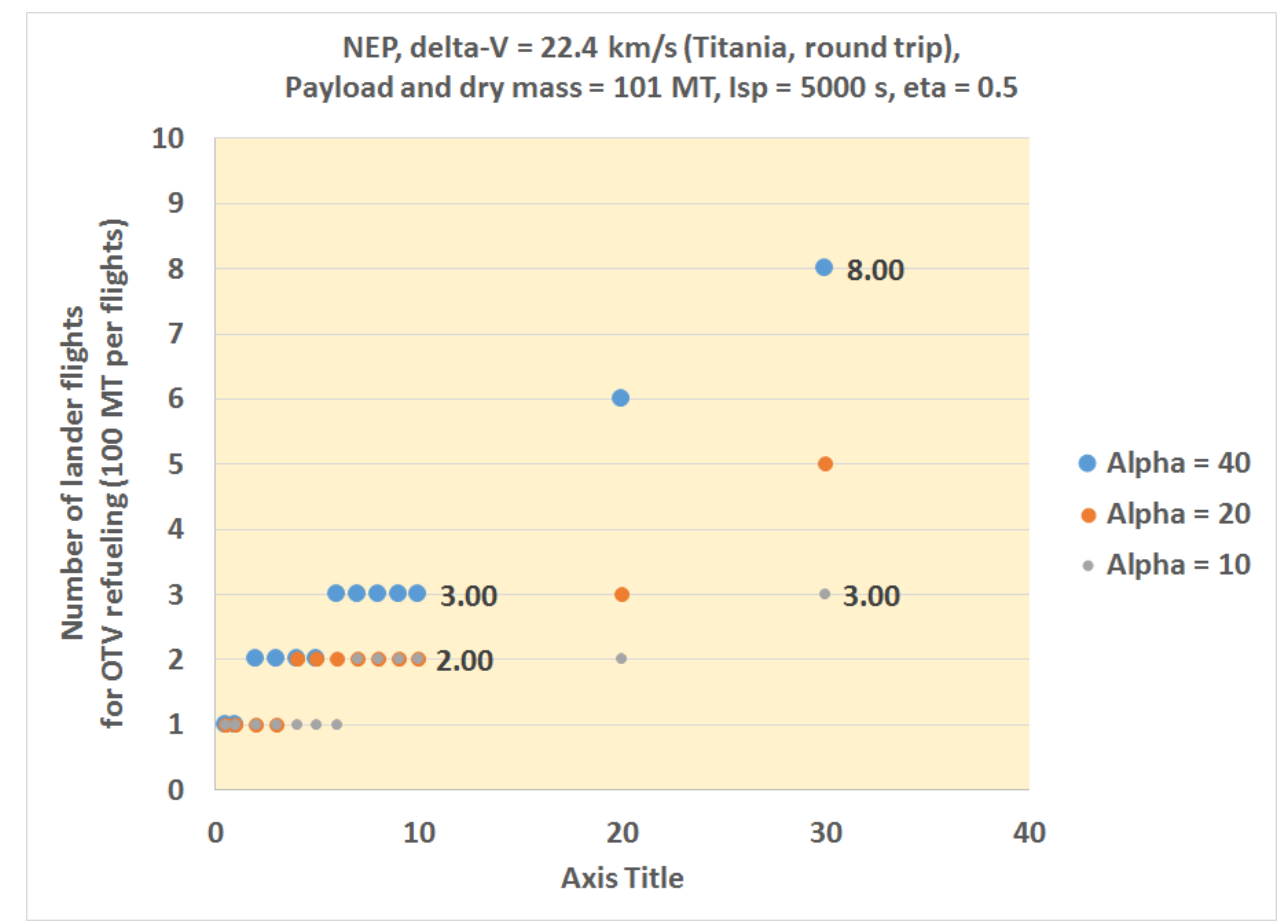

Figure A-1-12: Number of flights, 200 MT payloads - Titania OTVs

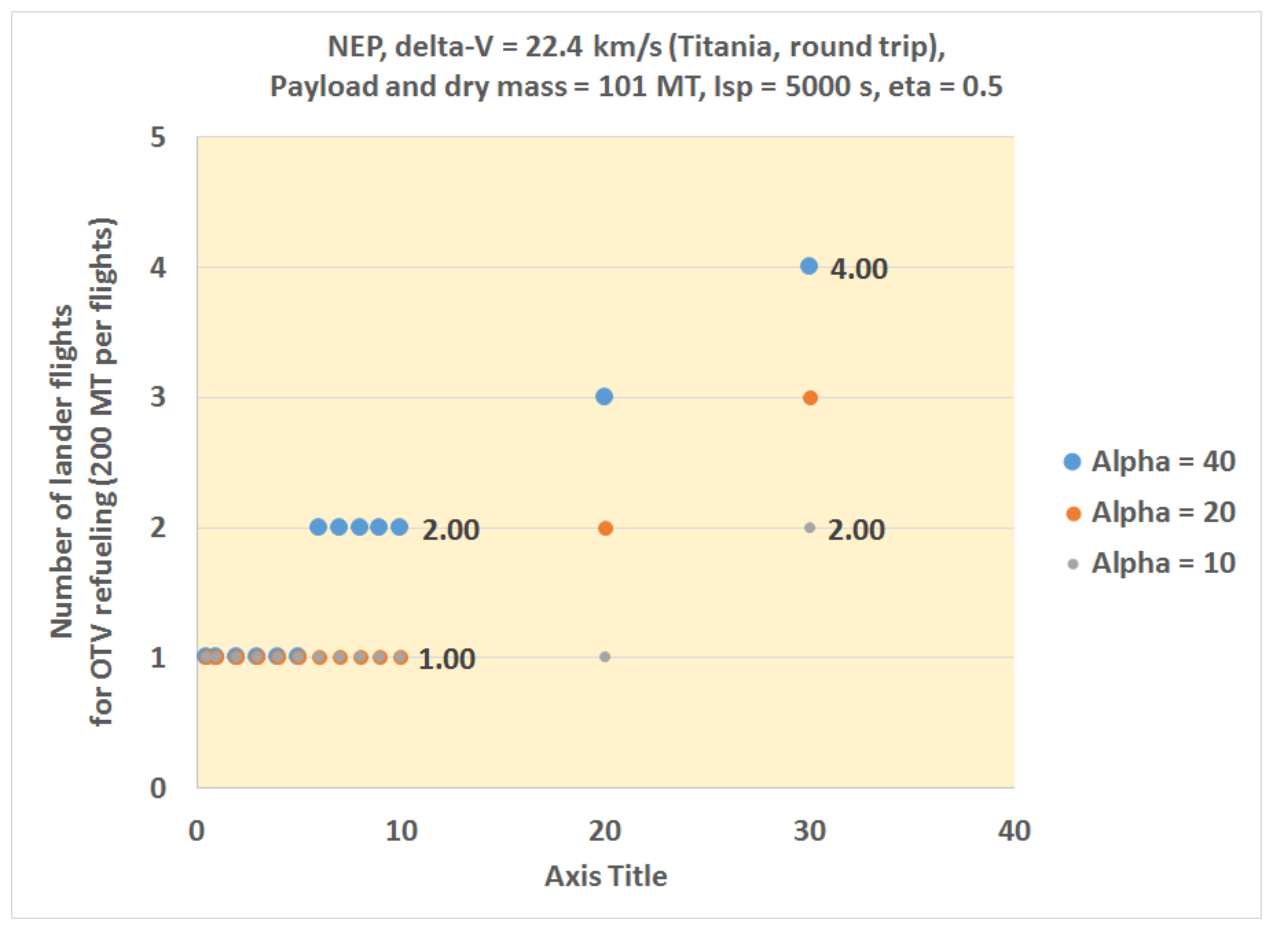


Figure A-1-13: Initial mass - Thalassa OTVs

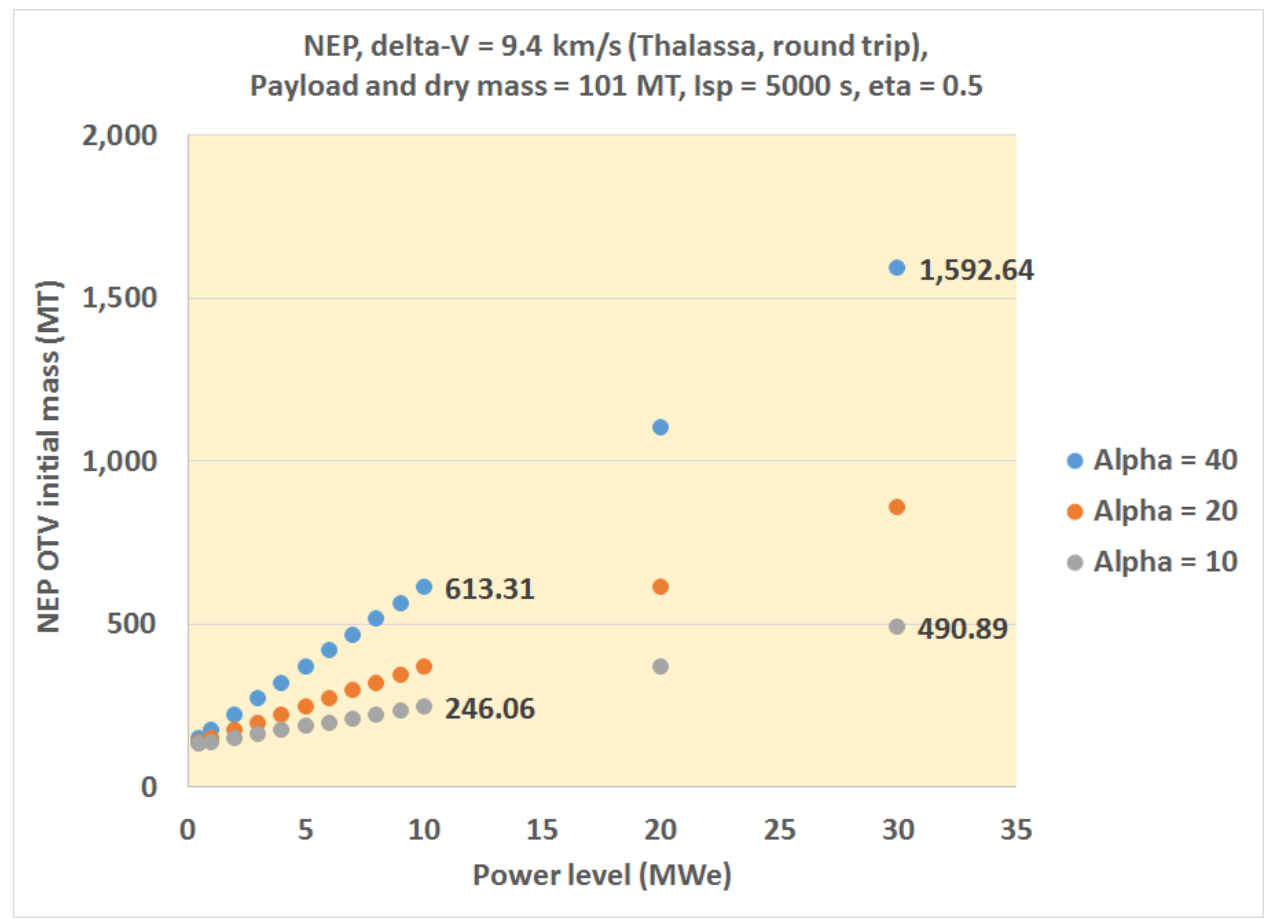

Figure A-1-14: Propellant mass - Thalassa OTVs

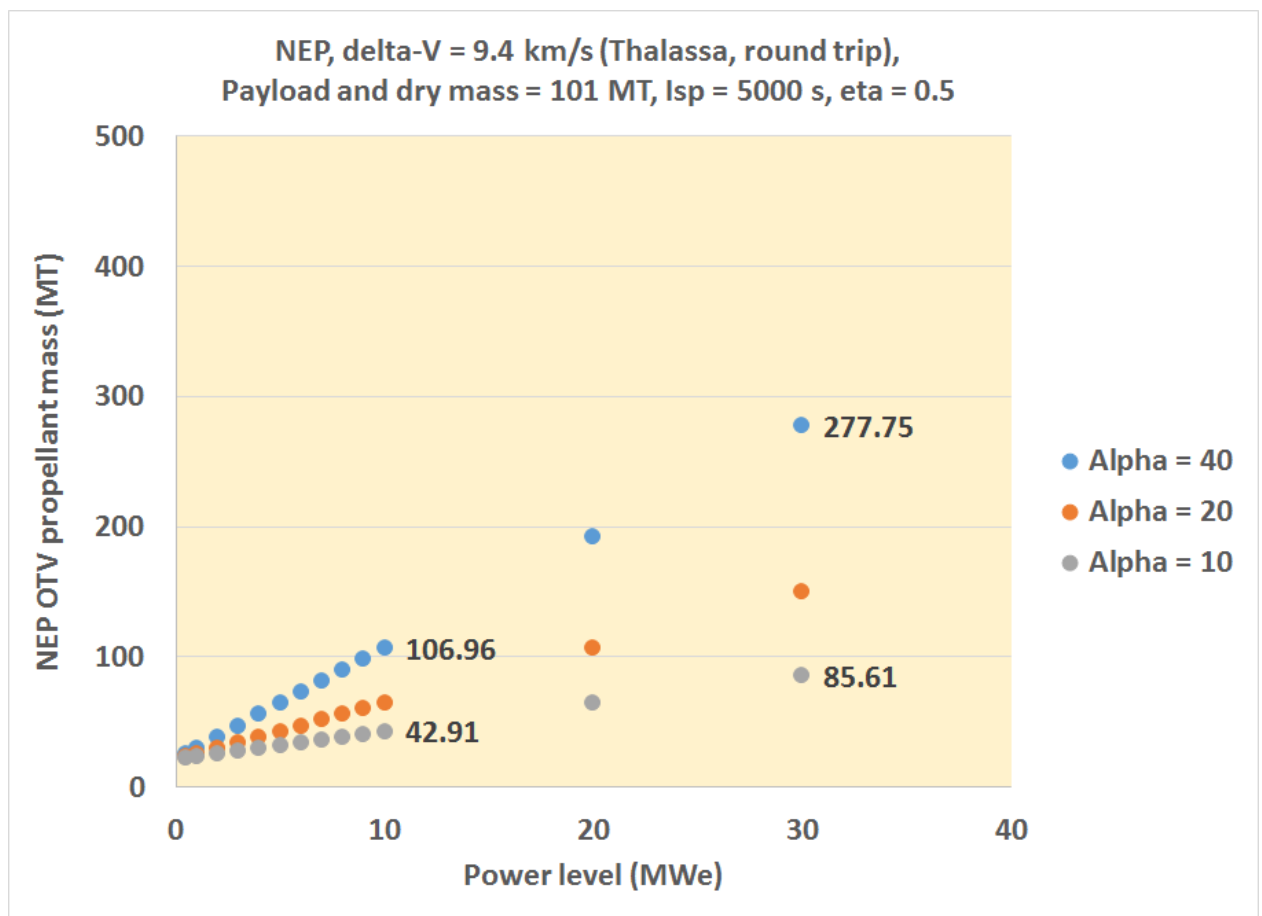


Figure A-1-15: Trip time - Thalassa OTVs

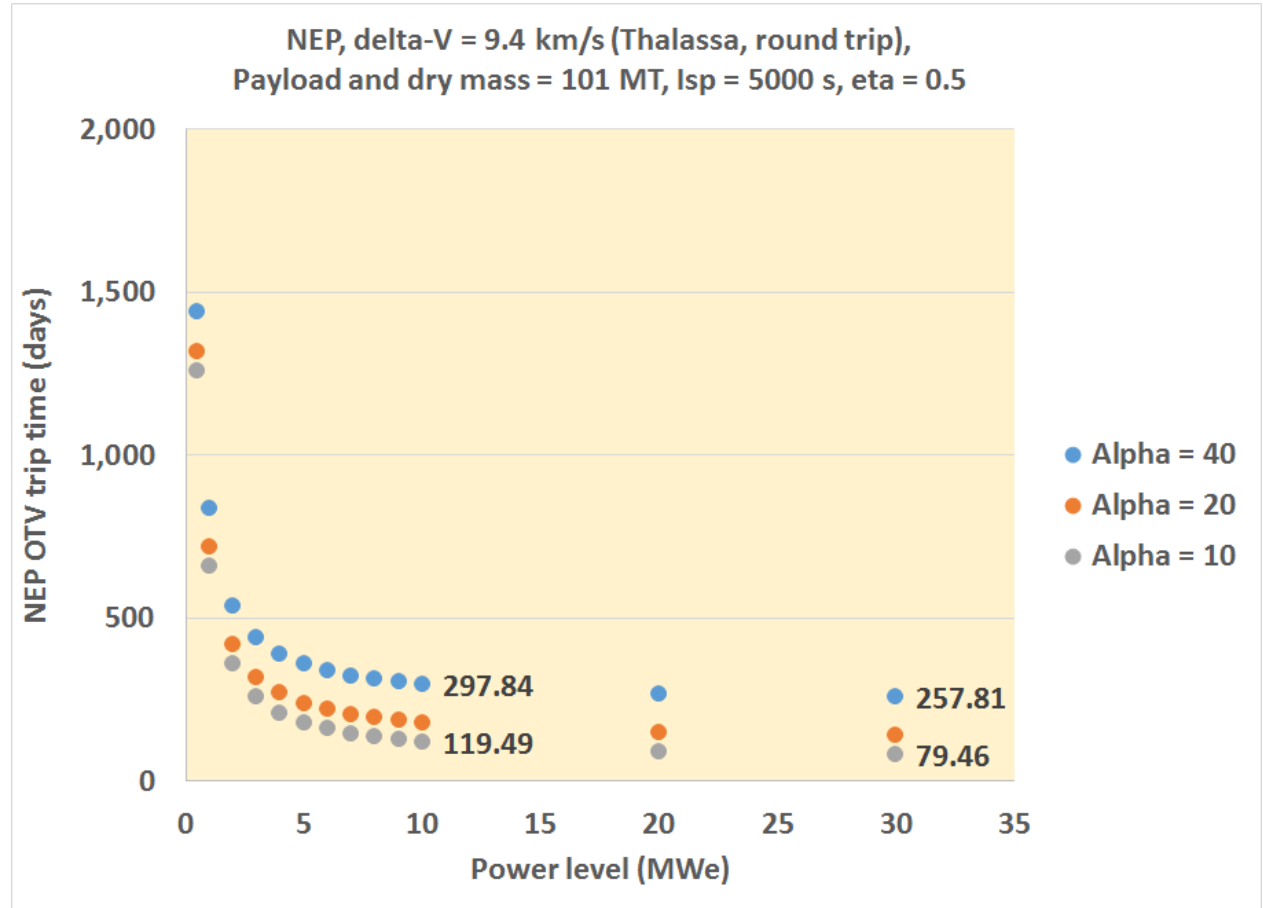

Figure A-1-16: Number of flights, 50 MT payloads - Thalassa OTVs

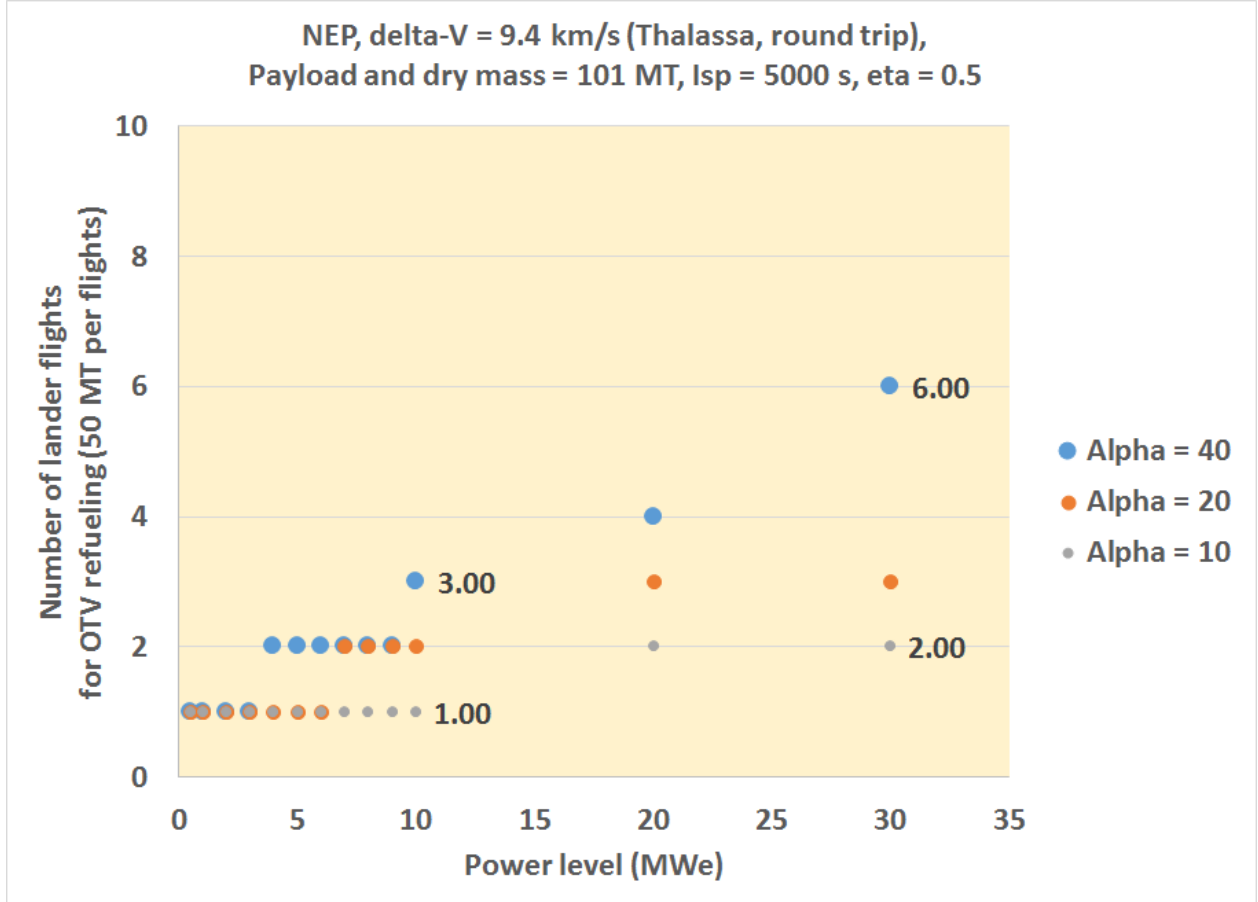


Figure A-1-17: Number of flights, 100 MT payloads - Thalassa OTVs

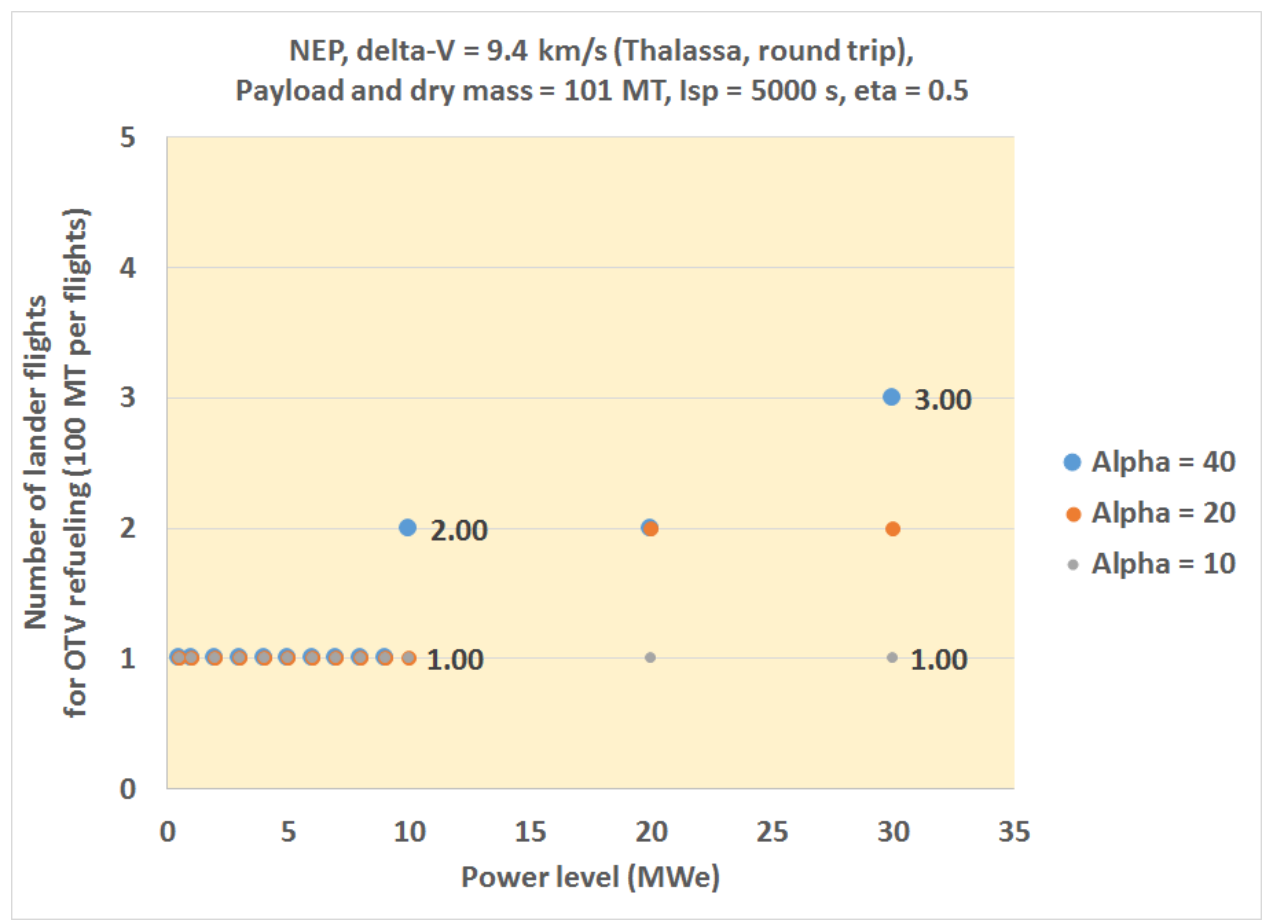

Figure A-1-18: Number of flights, 200 MT payloads - Thalassa OTVs

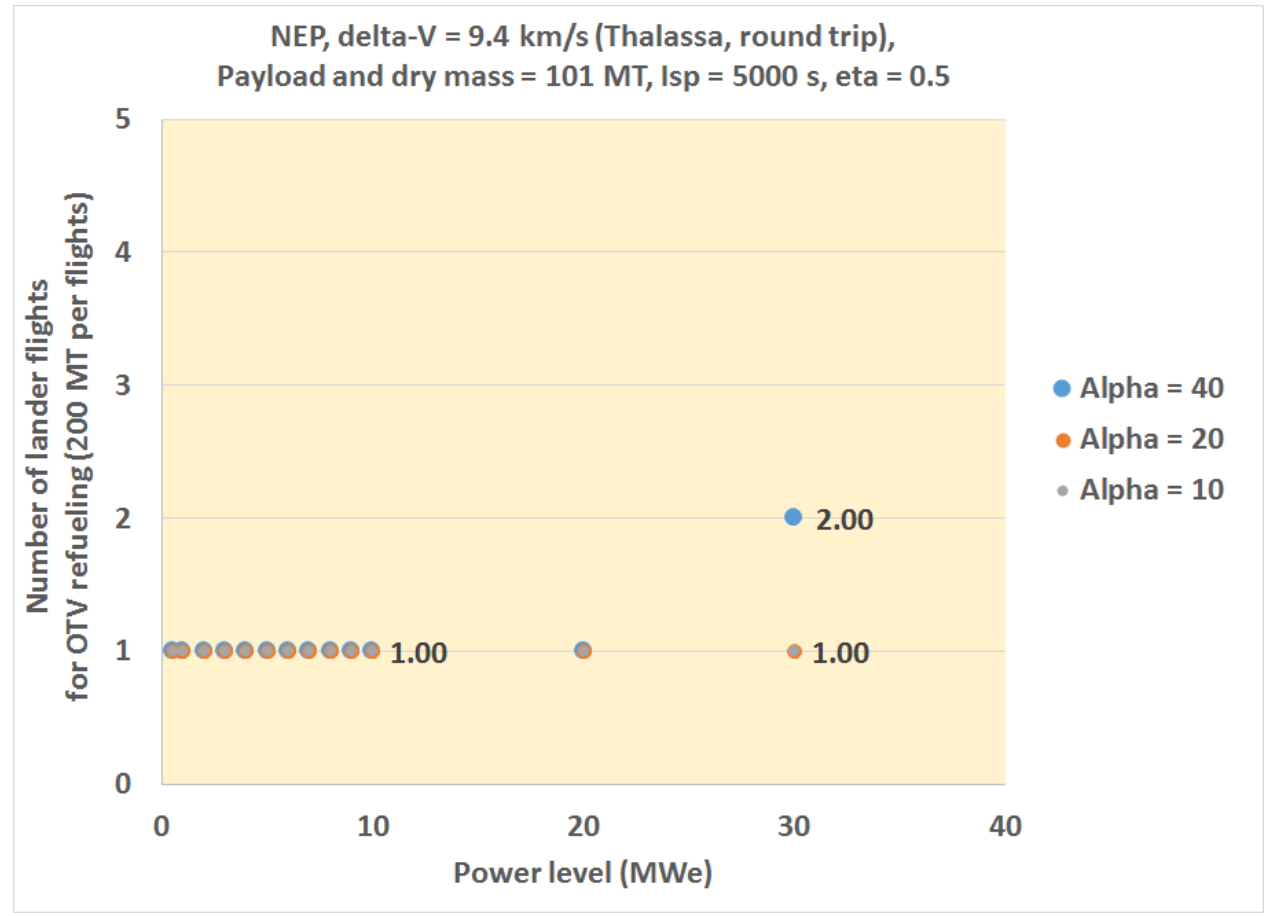


Figure A-1-19: Initial mass - Triton OTVs

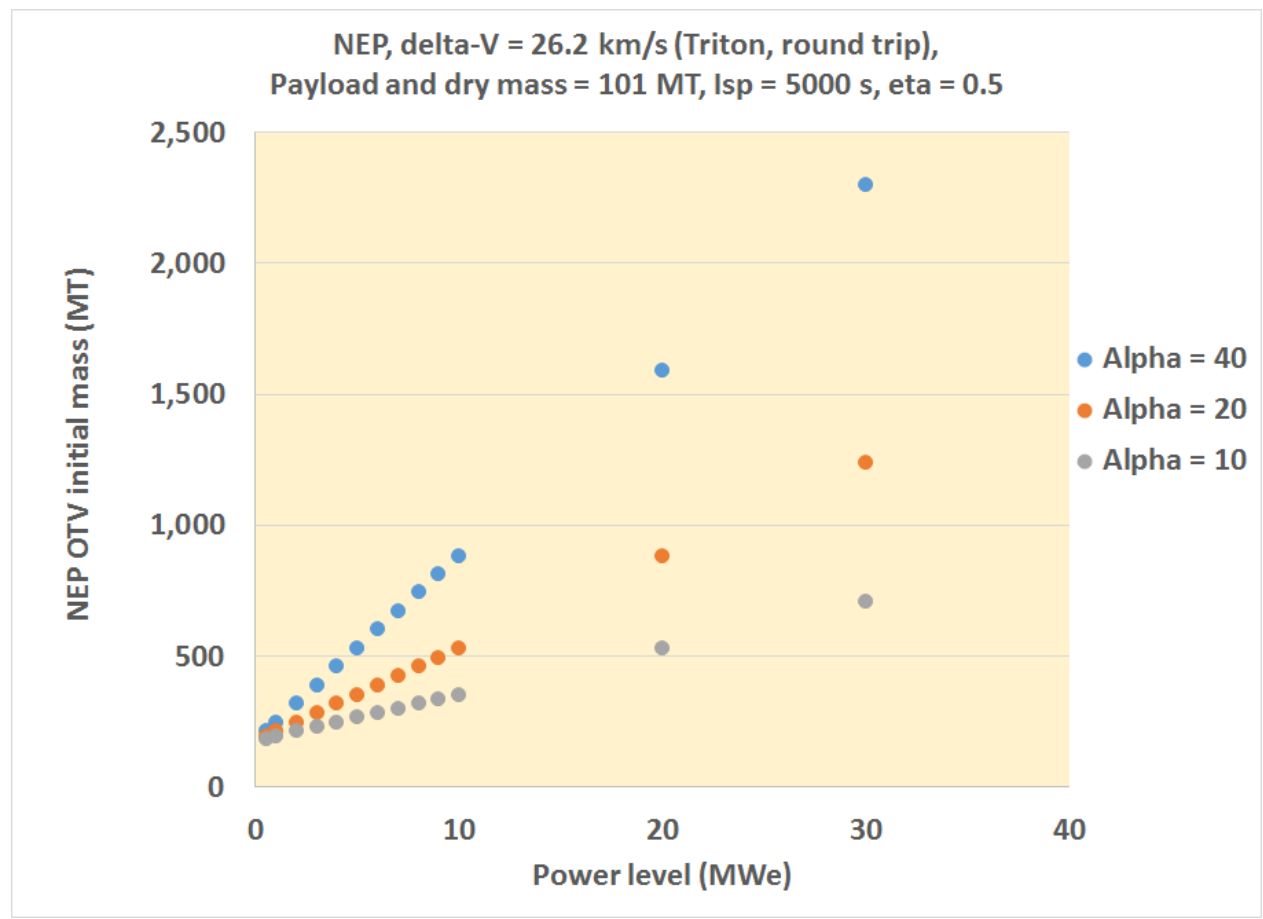

Figure A-1-20: Propellant mass - Triton OTVs

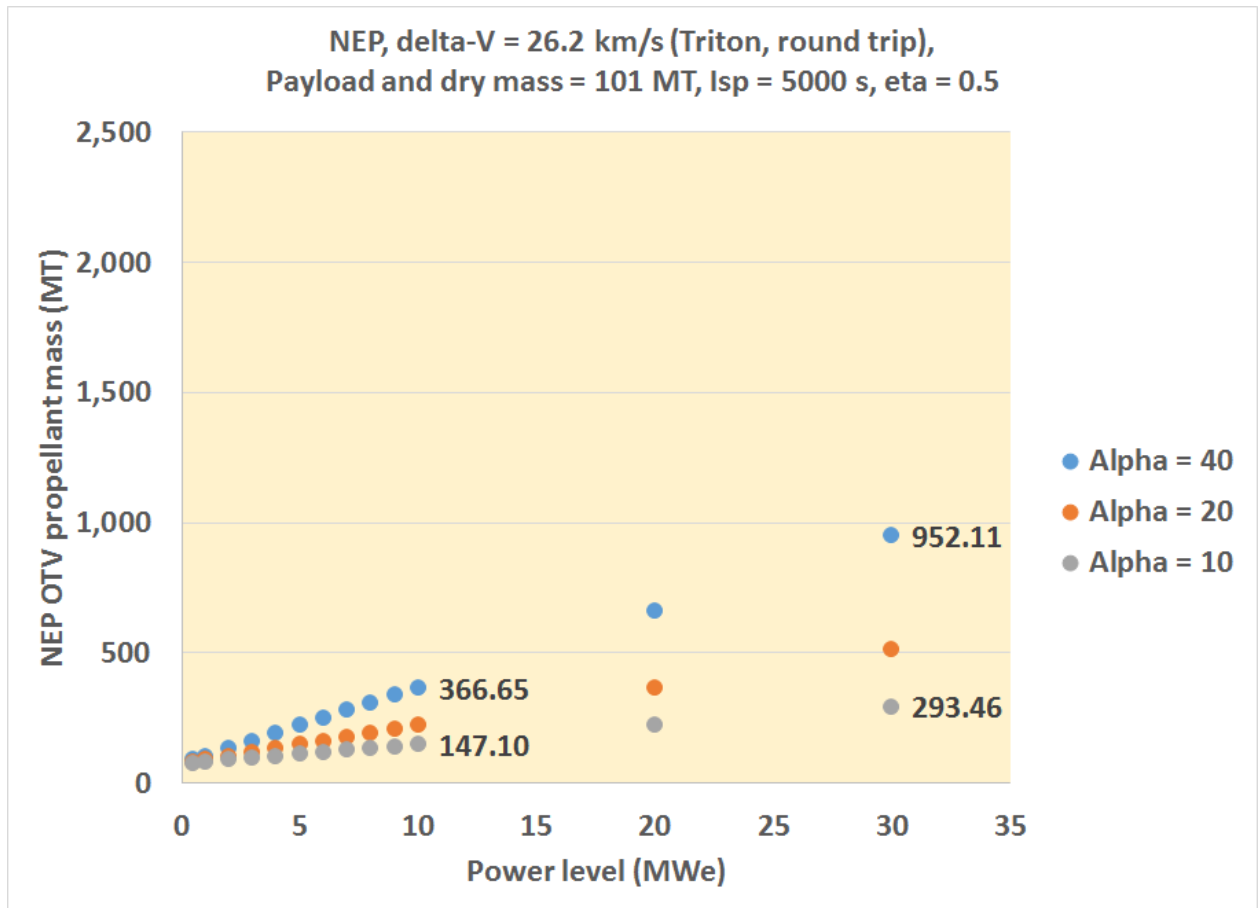


Figure A-1-21: Trip time - Triton OTVs

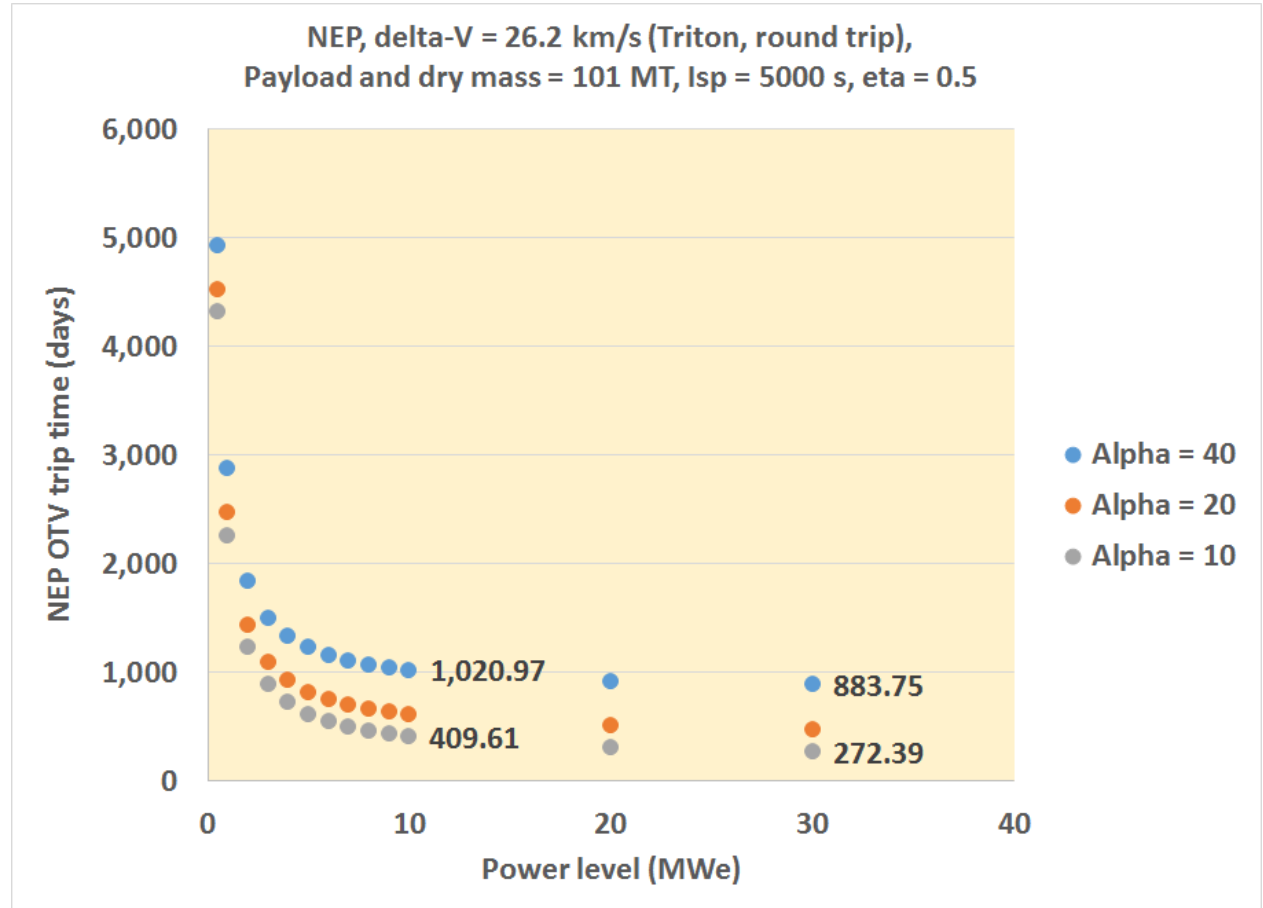

Figure A-1-22: Number of flights, 50 MT payloads - Triton OTVs

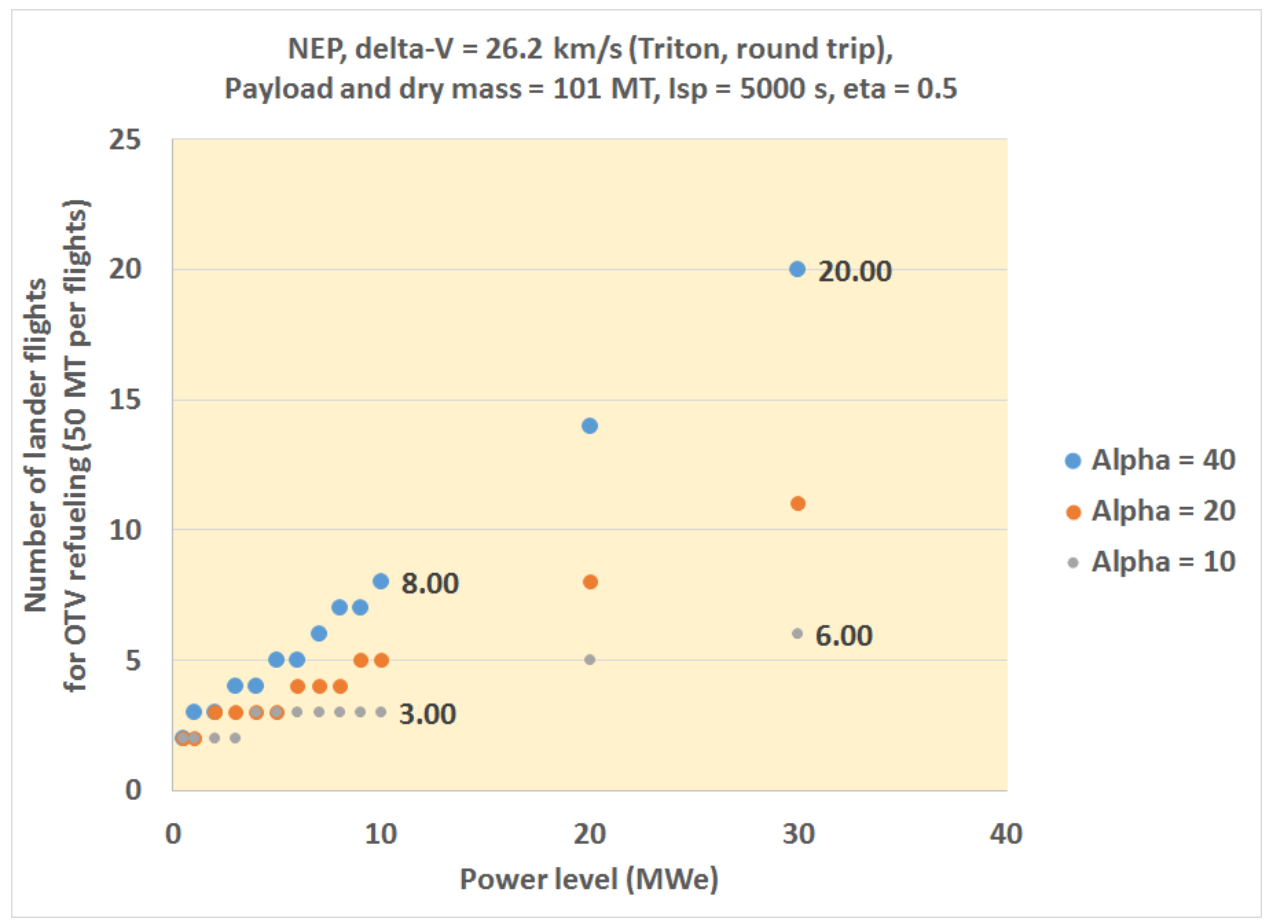


Figure A-1-23: Number of flights, 100 MT payloads - Triton OTVs

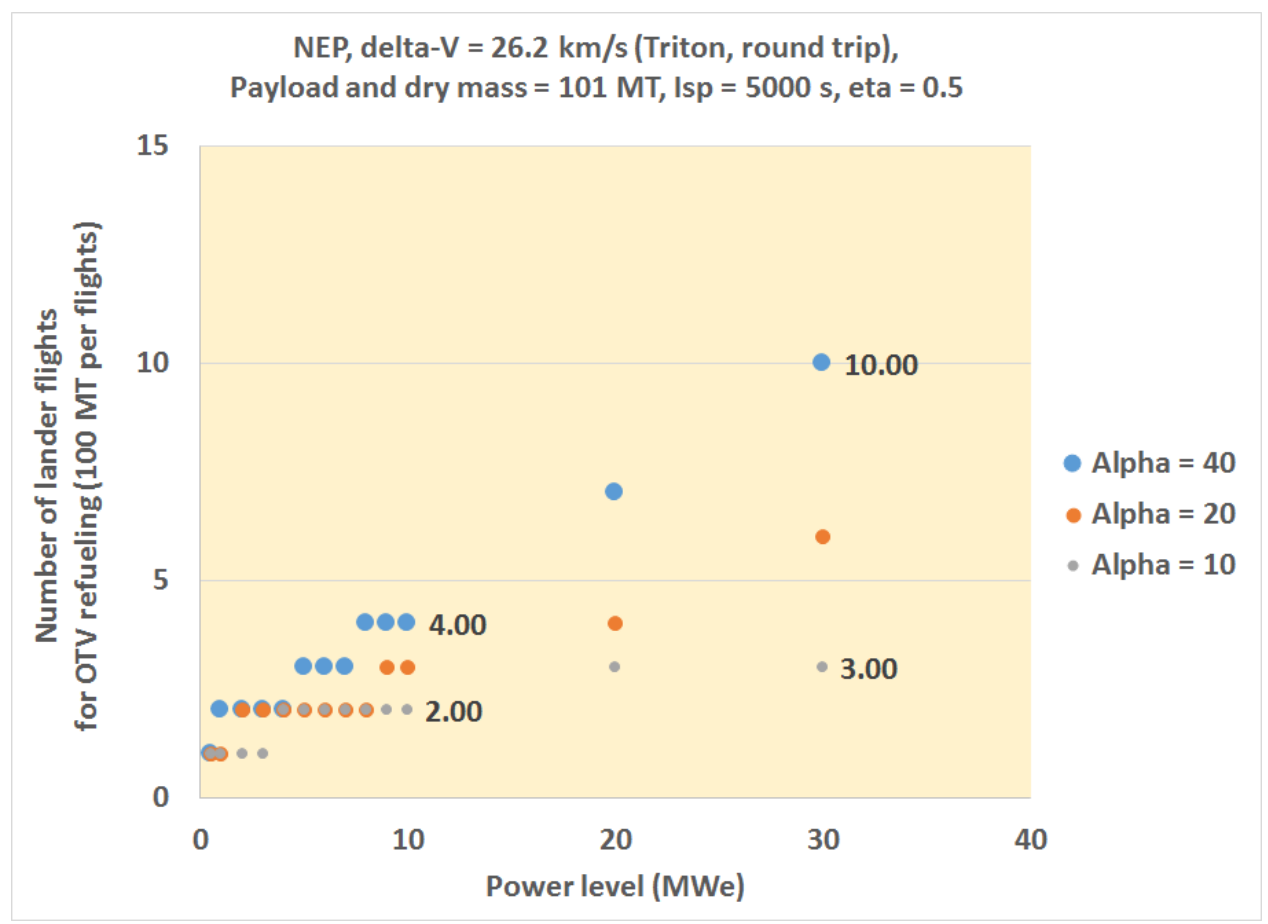

Figure A-1-24: Number of flights, 200 MT payloads - Triton OTVs

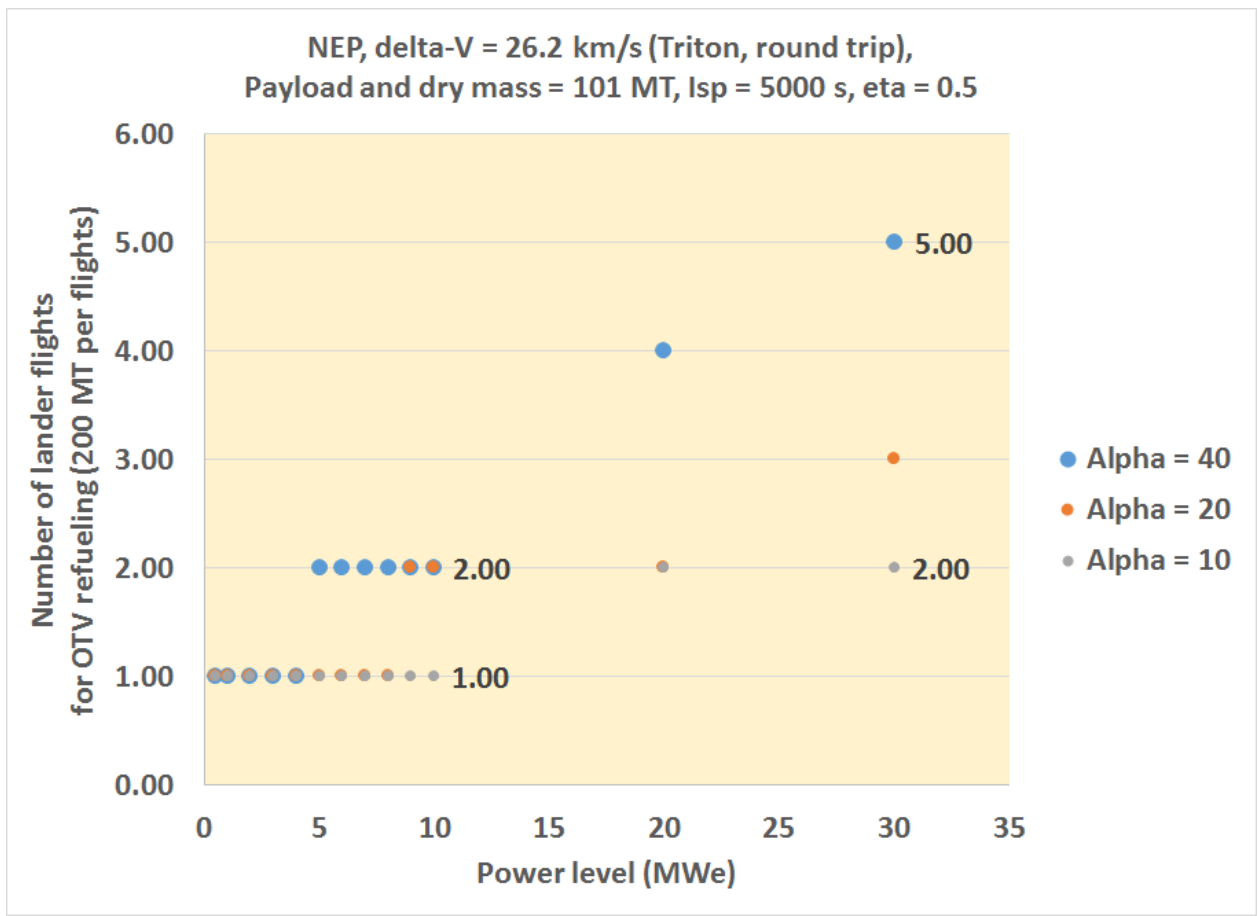




\section{Appendix A-2: AMOSS lander initial mass and propellant masses - for moon basing}

Figure A-2-1: Initial mass - Miranda lander

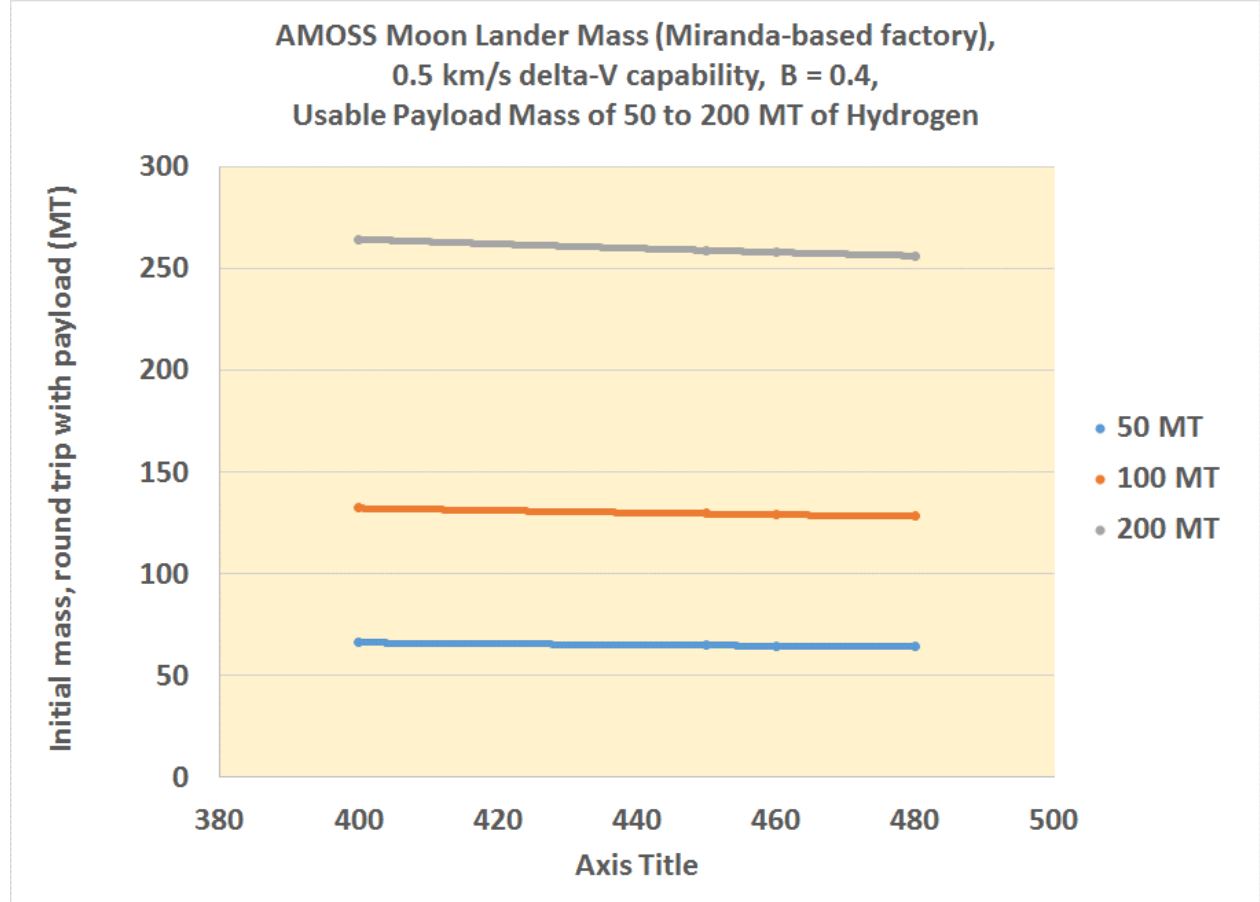

Figure A-2-2: Propellant mass - Miranda lander

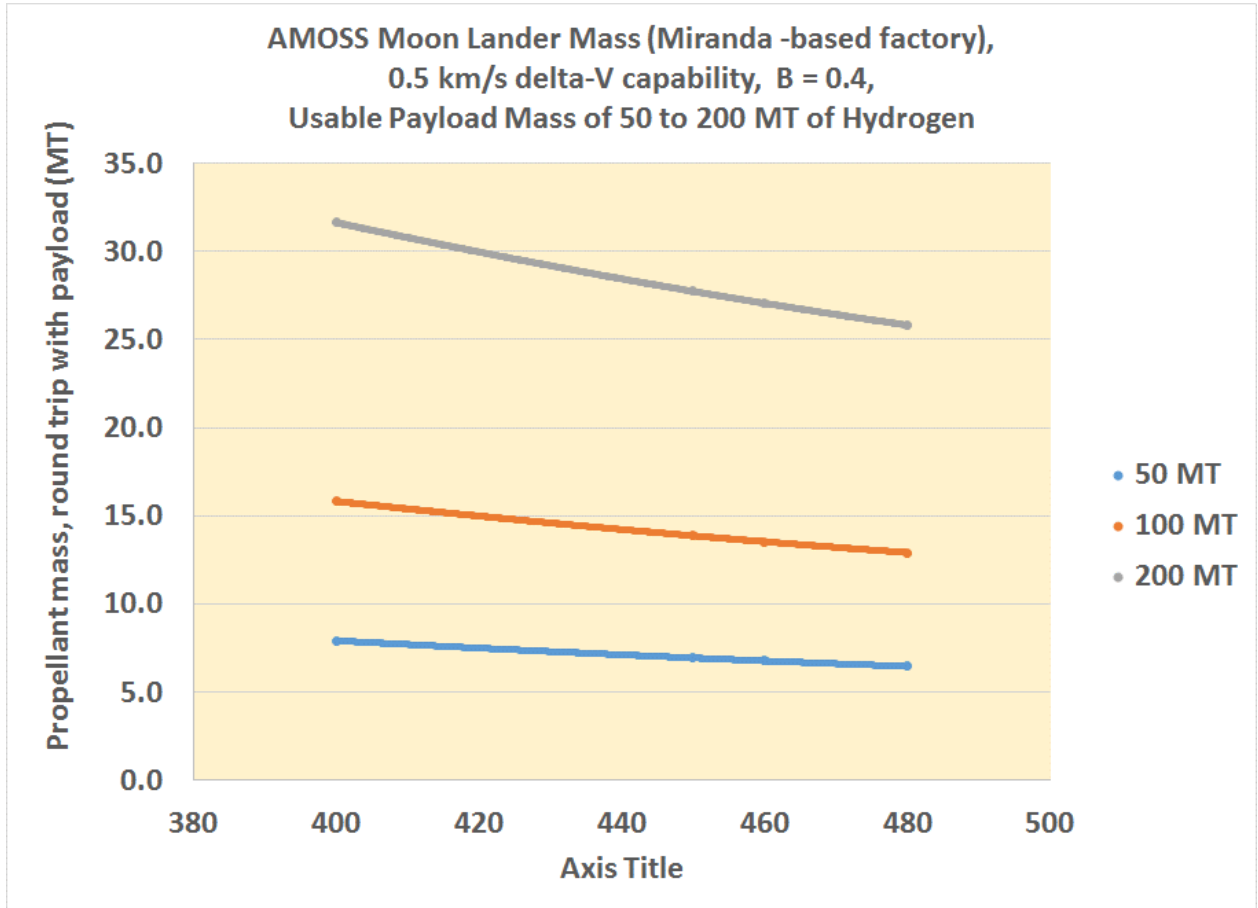

Figure A-2-3: Initial mass - Titania lander 


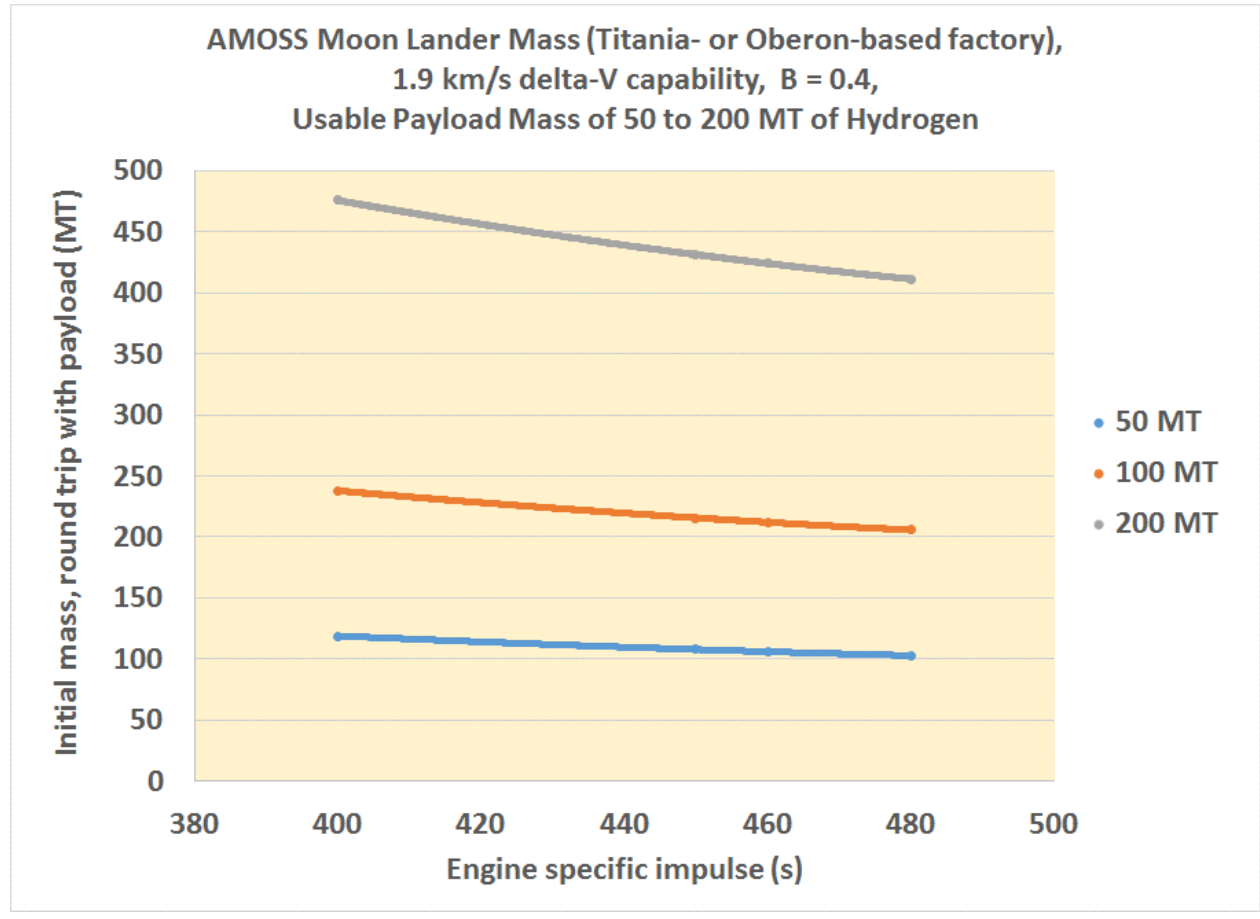

Figure A-2-4: Propellant mass - Titania lander

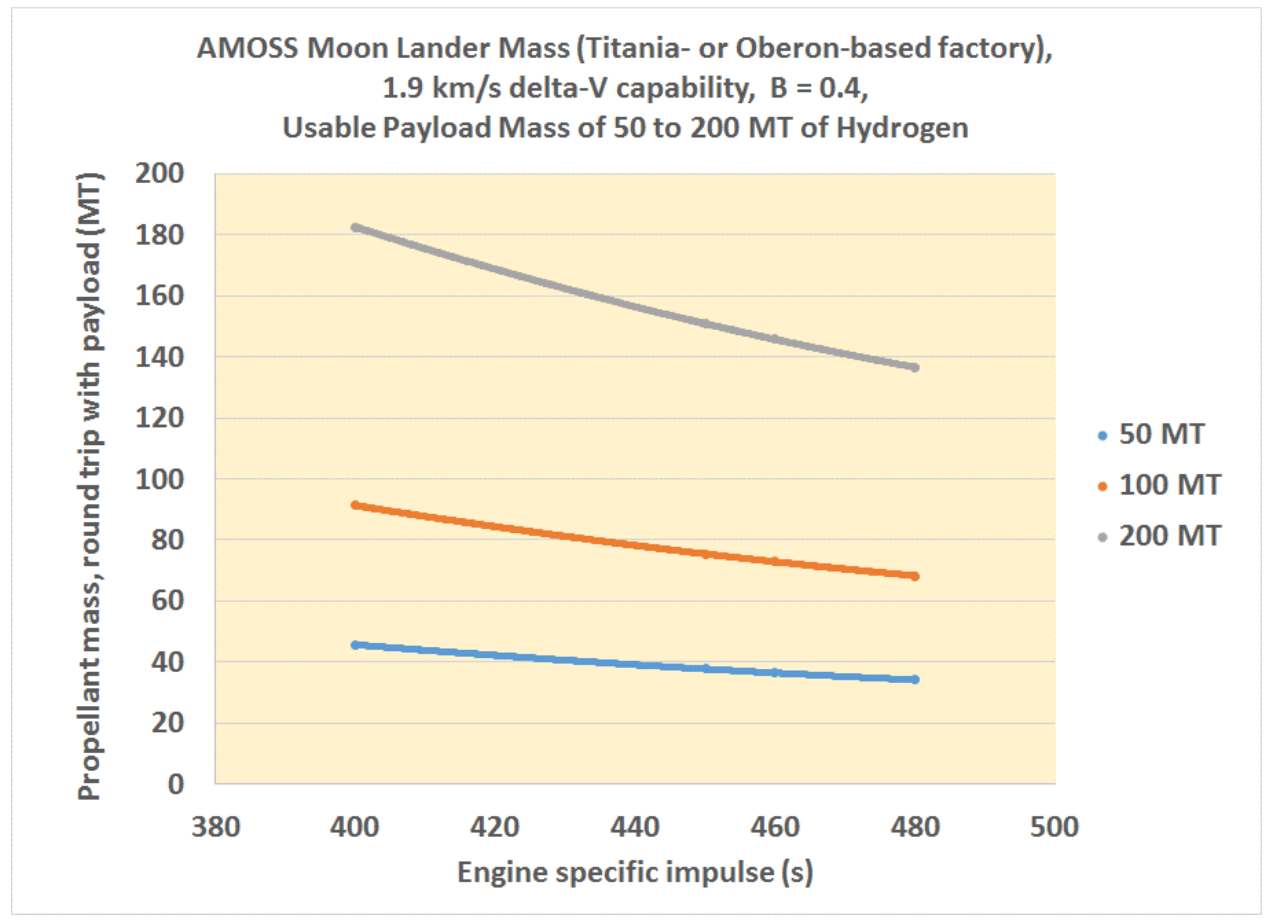


Figure A-2-5: Initial mass - Thalassa lander

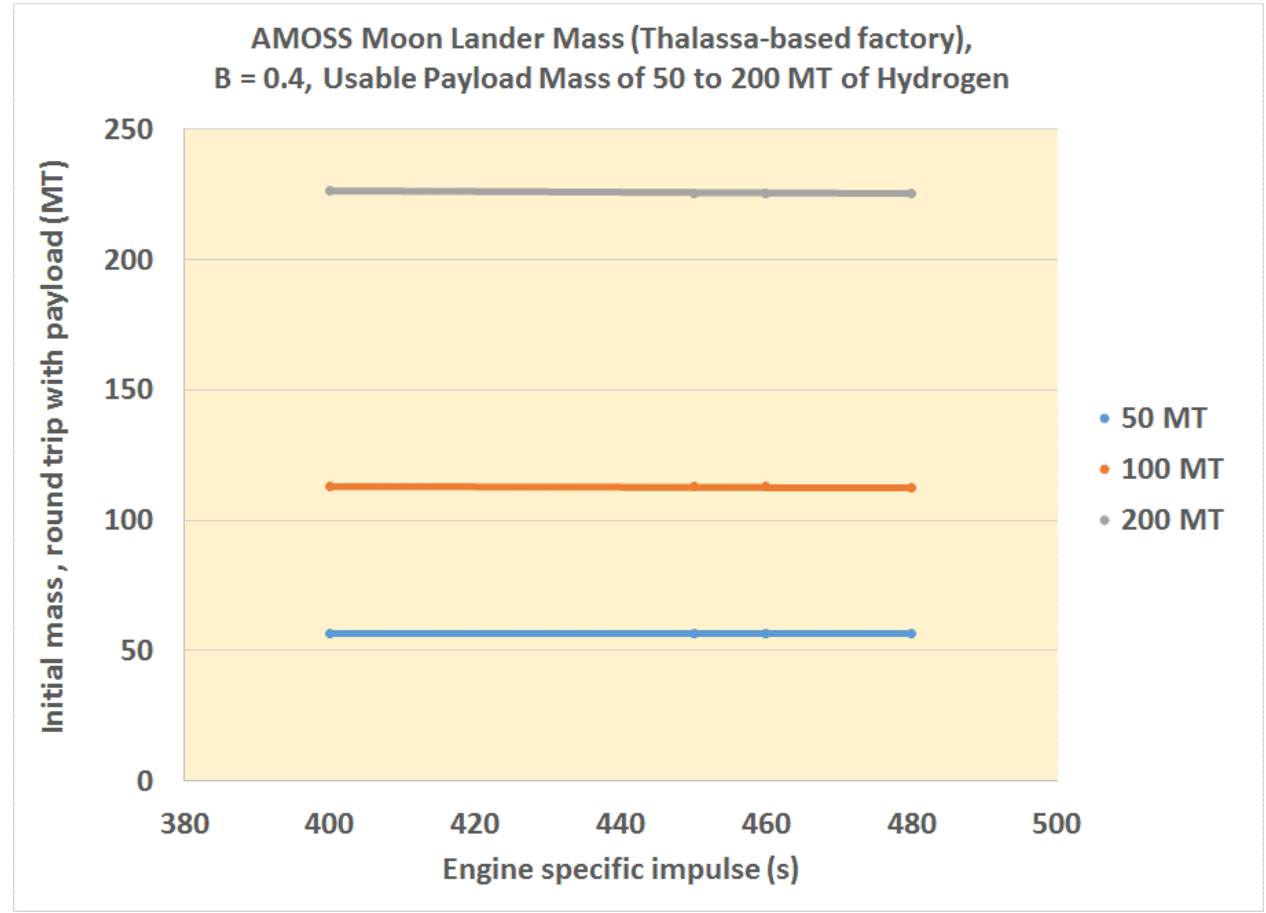

Figure A-2-6: Propellant mass - Thalassa lander

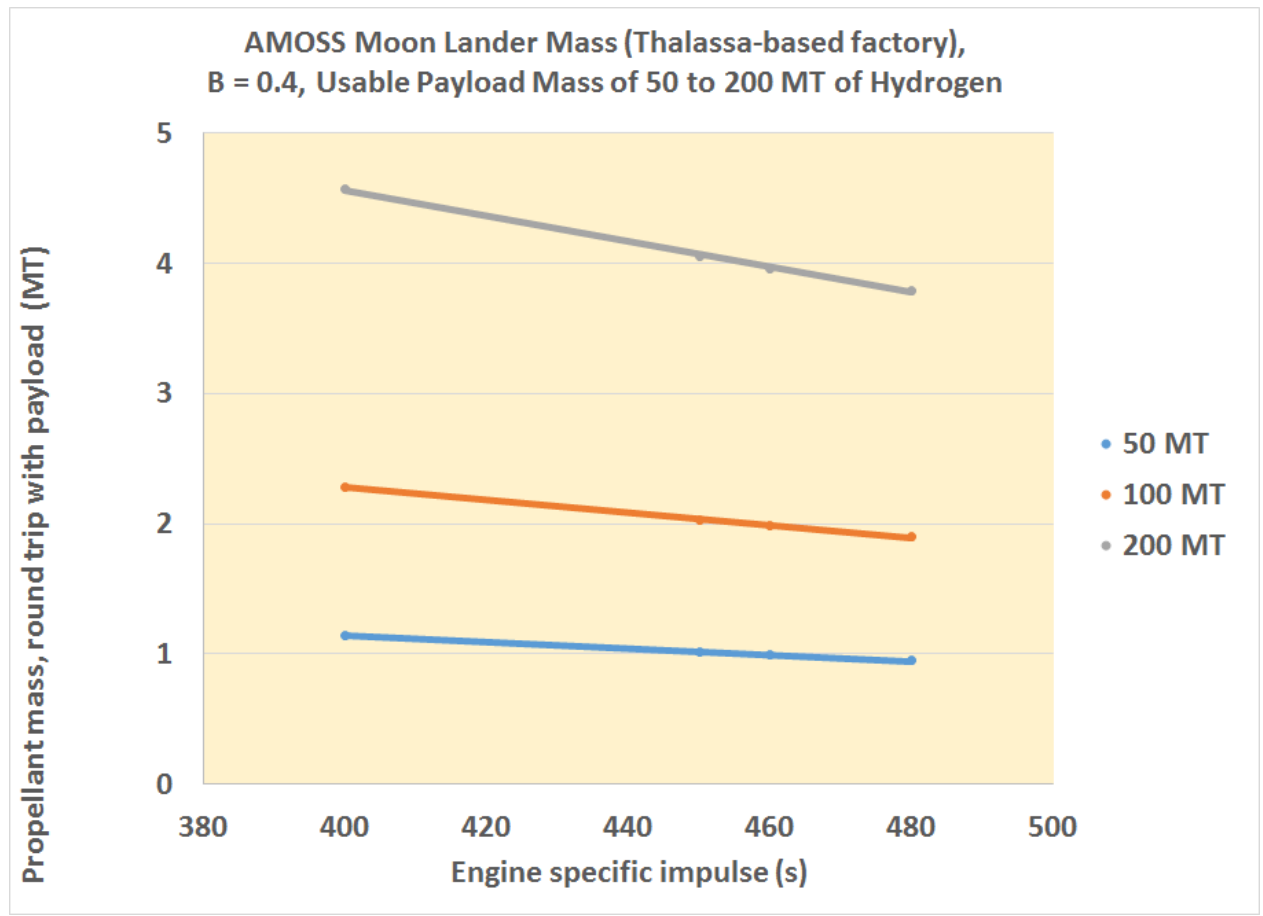


Figure A-2-7: Initial mass - Triton lander

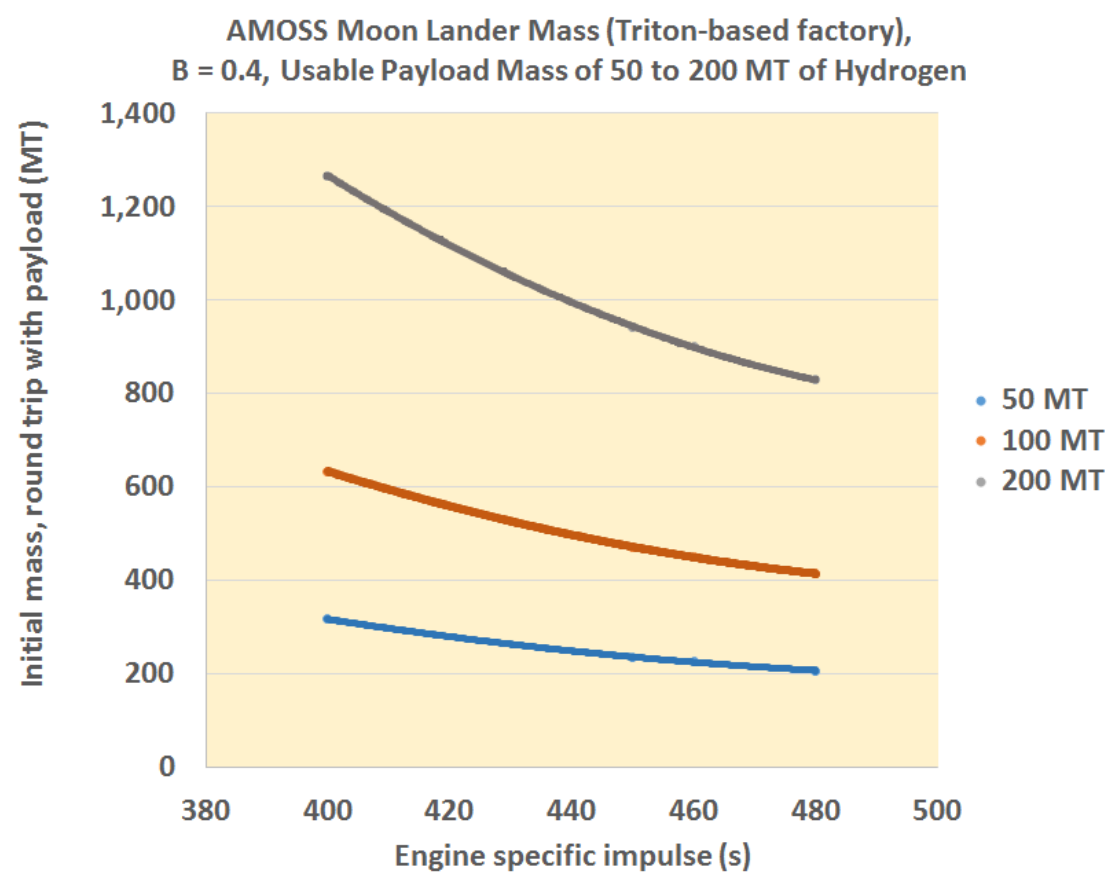

Figure A-2-8: Propellant mass - Triton lander

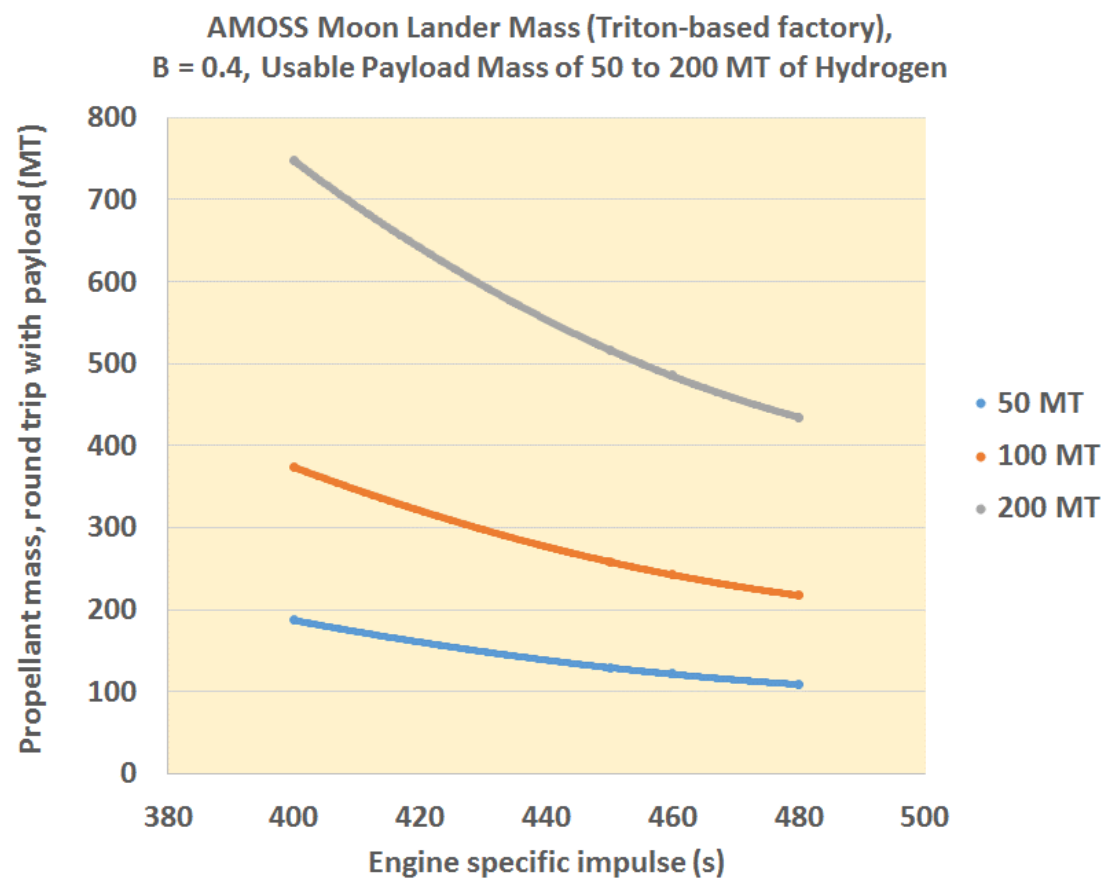




\section{Appendix B - Escape delta-V Values, one-way}

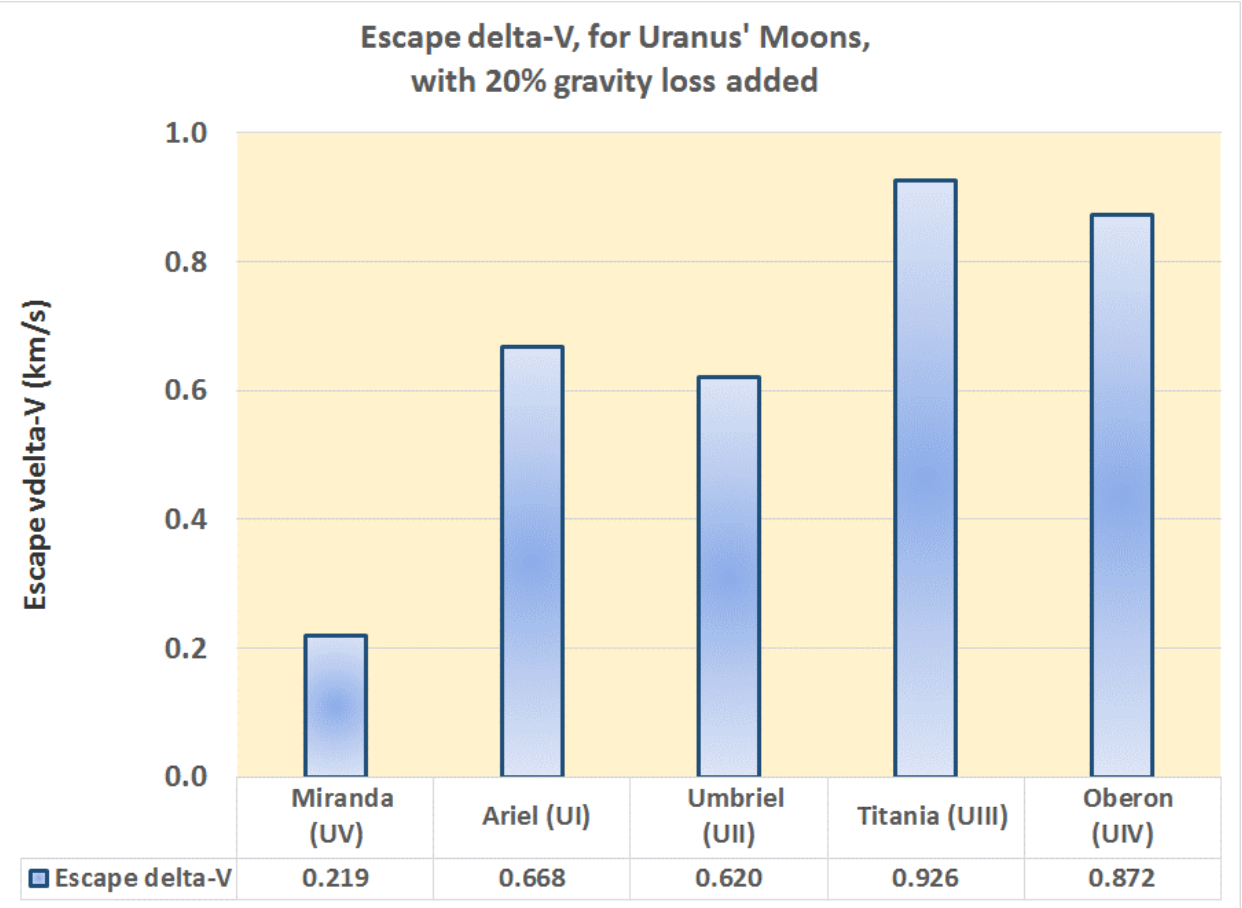

Figure B-1. Uranus' Moons - Escape delta-V (one way)

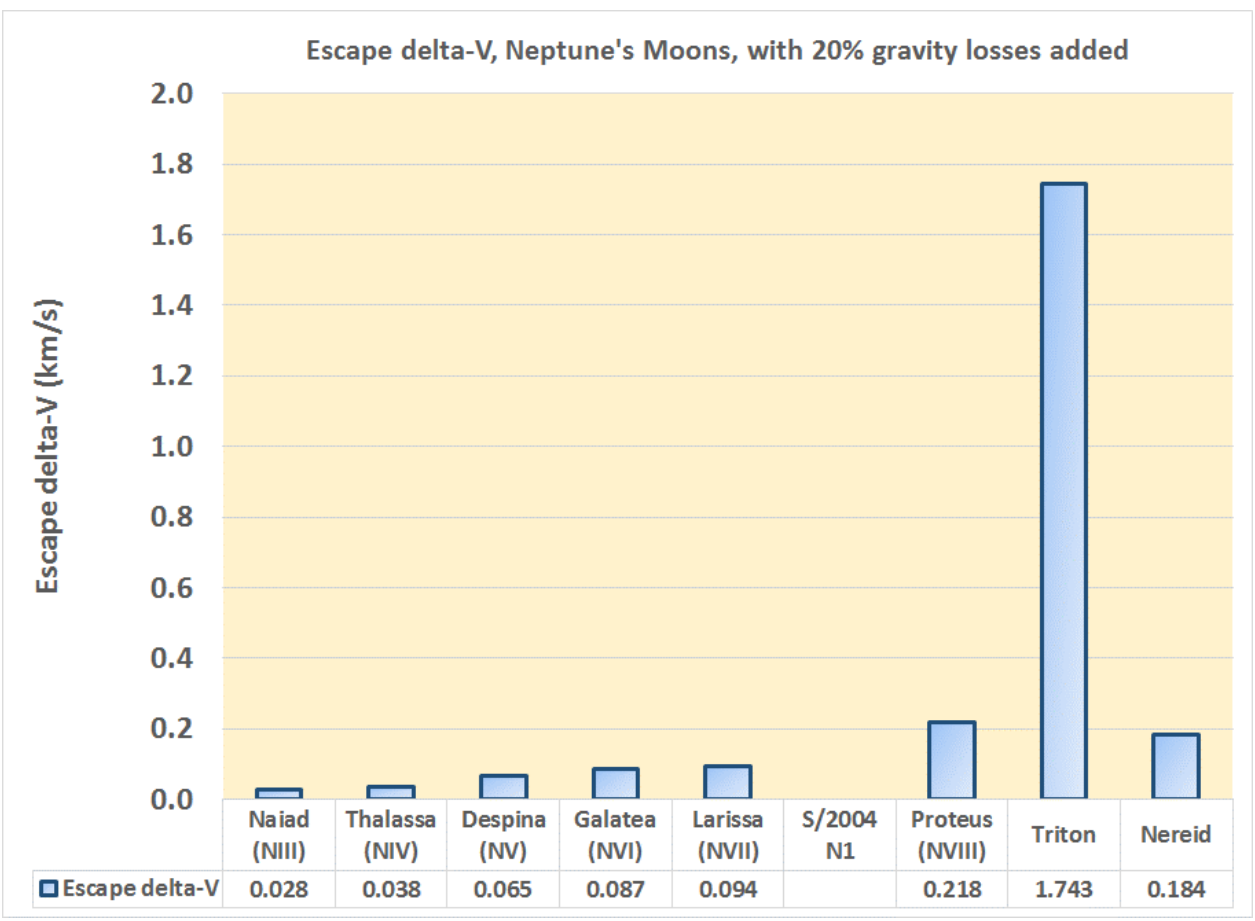

Figure B-2. Neptune's Moons - Escape delta-V (one way) 
Appendix C - Space Base Mass Estimates (based extrapolations of data in Refs. 37 and 38)

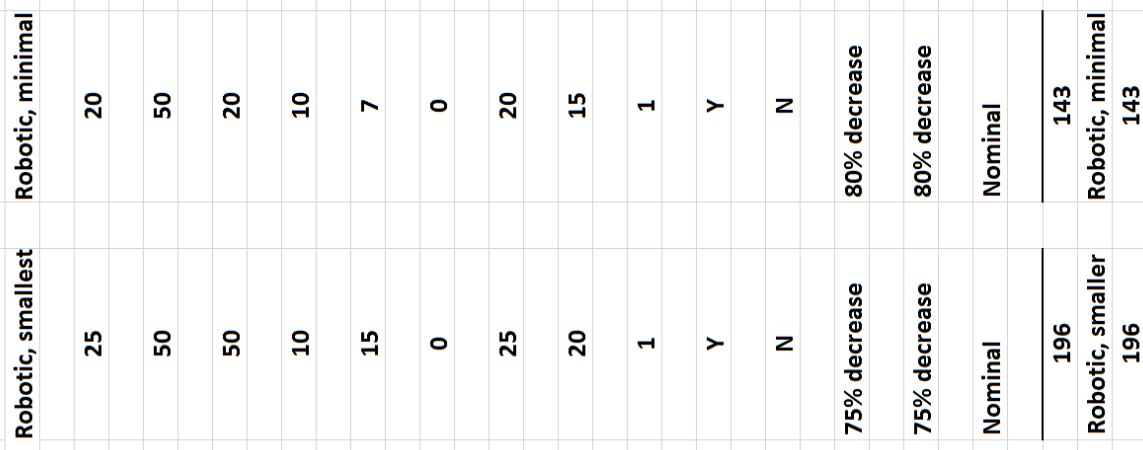

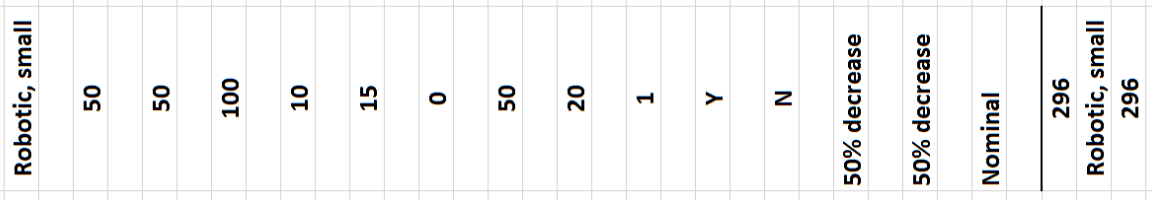

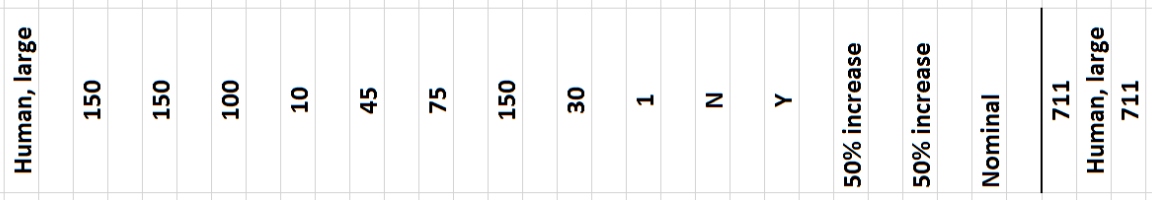

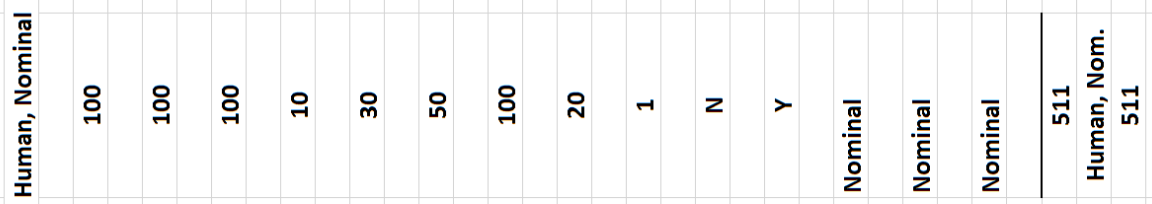

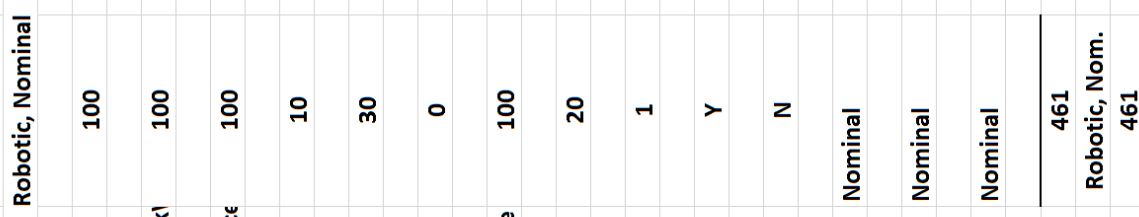

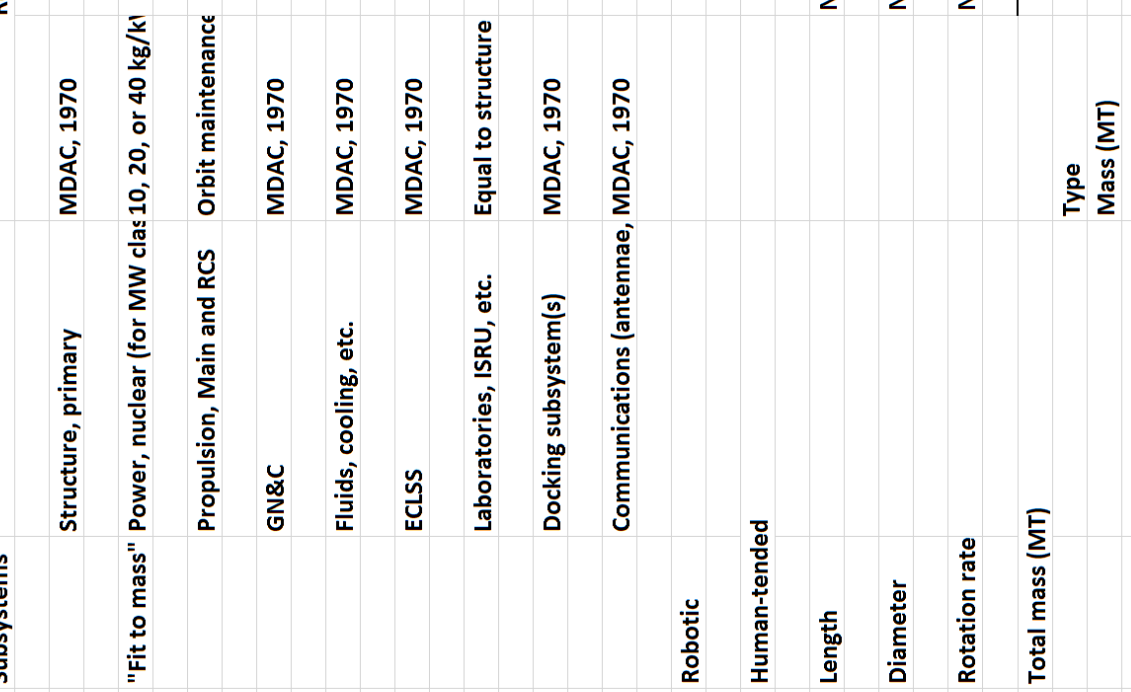




\section{References}

1) Palaszewski, B., "Atmospheric Mining in the Outer Solar System: Orbital Transfer Vehicles and Outer Planet Moon Base Options,” AIAA 2016-4889, July 2016.

2) Palaszewski, B., "Atmospheric Mining in the Outer Solar System: Aerial Vehicle Mission and Design Issues," AIAA 2015-4078, July 2015.

3) Palaszewski, B., "Atmospheric Mining in the Outer Solar System: Aerial Vehicle Reconnaissance and Exploration Options," AIAA 2014-3819, July 2014.

4) Palaszewski, B., "Atmospheric Mining in the Outer Solar System: Resource Capturing, Exploration, and Exploitation,” AIAA 2013-3765, August 2013.

5) Palaszewski, B., "Atmospheric Mining in the Outer Solar System: Resource Capturing, Storage, and Utilization," AIAA 2012-3742, August 2012.

6) Palaszewski, B., "Atmospheric Mining in the Outer Solar System: Issues and Challenges for Mining Vehicle Propulsion,” AIAA 2011-6041, August 2011.

7) Palaszewski, B., “Atmospheric Mining in the Outer Solar System: University Studies of Mining Vehicles and Propulsion," AIAA 2010-6573, August 2010.

8) Palaszewski, B., "Atmospheric Mining in the Outer Solar System: Mining Design Issues and Considerations," AIAA 2009-4961, August 2009.

9) Palaszewski, B., "Atmospheric Mining in the Outer Solar System: Orbital Transfer Vehicles and Outer Planet Moon Base Options," AIAA 2008-4861, July 2008.

10) Palaszewski, B., "Atmospheric Mining in the Outer Solar System: Mission Scenarios and Options for In-Situ Resource Utilization.” AIAA 2007-5598, July 2007.

11) Palaszewski, B., “Atmospheric Mining in the Outer Solar System: Vehicle Sizing Issues.” AIAA 2006-5222, July 2006.

12) Palaszewski, B., ”Atmospheric Mining in the Outer Solar System,” AIAA 2005-4319, July 2005.

13) R. Frisbee, “Advanced Space Propulsion for the 21st Century," Journal of Propulsion and Power, Vol. 19, No. 6, Nov-Dec 2003.

14) Dunn, Bruce P., "High-Energy Orbit Refueling for Orbital Transfer Vehicles," Journal of Spacecraft and Rockets Volume. 24, No. 6, 1987, pp. 518-522.

15) Noca, M.; Polk, J. E. "Ion Thrusters And LFAs For Outer Planet Exploration, “ IAF 6th International Symposium; Versailles; France, May 2002.

16) Hunt, James L., Laruelle, Gerard, Wagner, Alain, “Systems Challenges for Hypersonic Vehicles;” AGARD Interpanel Symposium on Future Aerospace Technology in Service to the Alliance, NASA-TM-112908, AGARDPaper-C37, 1997.

17) Starr, Brett R.; Westhelle, Carlos H.; Masciarelli, James P., "Aerocapture Performance Analysis For A NeptuneTriton Exploration Mission,” NASA/TM-2006-214300, April 2006. 
18) Bussard, R., “ASPEN II: Two Staging and Radiation Shielding Effects on ASPEN Vehicle Performance," LA26-80, 09/06/1967 and in "ASPEN: Nuclear Propulsion for Earth to Orbit Aerospace Plane Vehicles," Robert W. Bussard, Proceedings International Conference on Spaceflight, Rome. June 1971.

19) Bussard, R., and Jameson, L.W.; "The QED Engine Spectrum: Fusion-Electric Propulsion for Airbreathing to Interstellar Flight," AIAA paper 93-2006, 29th Joint Propulsion Conference, Monterey, CA 6/28 to 6/30/1993; in JPP, Volume 11, Number 2, pp 365/372.

20) Borowski, Stanley K.; Dudzinski, Leonard A.; and McGuire, Melissa L.: "Artificial Gravity Vehicle Design Option for NASA’s Human Mars Mission Using “Bimodal” NTR Propulsion,” AIAA Paper 99-2545, 1999.

21) Kendall, J. S.; Stoeffler, R. C., "Conceptual design studies and experiments related to cavity exhaust systems for nuclear light bulb configurations," Report Number: L-910900-15; NASA-CR-129298

22) Latham, T. S.; Rodgers, R. J., "Small nuclear light bulb engines with cold beryllium reflectors," Report Number: AIAA PAPER 72-1093.

23) Latham, T. S., "Summary of the performance characteristics of the nuclear light bulb engine," Report Number: AIAA PAPER 71-642.

24) Rodgers, R. J.; Latham, T. S., "Analytical design and performance studies of the nuclear light bulb engine," Report Number: L-910900-16; NASA-CR-129295.

25) RONALD GREELEY, et al., "A Scientific Rationale for Mobility in Planetary Environments," Committee on Planetary and Lunar Exploration, National Research Council, NATIONAL ACADEMY PRESS, Washington, D.C. 1999.

26) Herbert, F. and Sandel, B.R., "Ultraviolet observations of Uranus and Neptune," Planetary and Space Science Vol. 47, pp. 1119 to 1139, Published by Elsevier Science Ltd.

27) Sromovsky, L.A., et al., "Episodic bright and dark spots on Uranus” Icarus, Vol. 220 (2012), pp. 6-22.

28) K.A. Rages, H.B. Hammel, A.J. Friedsond, "Evidence for temporal change at Uranus' south pole,” Icarus, Vol. 172 (2004), pp. 548-554).

29) L. A. Sromovsky and P. M. Fry, et al. "Coordinated 1996 HST and IRTF Imaging of Neptune and Triton III: Neptune's Atmospheric Circulation and Cloud Structure,” Icarus, Vol. 149 (2001)., pp. 459-488.

30) Turba, R.D., "DESIGN OF A NUCLEAR PROPULSION SYSTEM FOR AN UNMANNED AERIAL VEHICLE," Vandebilt University, Masters Thesis, May 2011.

31) Maise, George; Powell, James; Paniagua, John; Ludewig, Hans; Todosow, Michael, "Exploration of Jovian atmosphere using nuclear ramjet flyer," IAF Paper 98-S608, 1998.

32) Borowski, S., "Human lunar mission capabilities using SSTO, ISRU and LOX-augmented NTR technologies A preliminary assessment," AIAA 1995-0026, 1995

33) Robert H. Frisbee* and Ioannis G. Mikellides, "The Nuclear-Electric Pulsed Inductive Thruster (NuPIT): Mission Analysis for Prometheus," AIAA 2005-3892, July 2005.

34) Grundy, W.M., et al., "Distributions of $\mathrm{H} 2 \mathrm{O}$ and $\mathrm{CO} 2$ ices on Ariel, Umbriel, Titania, and Oberon from IRTF /SpeX observations," Icarus, 184, (2006), 543-555.

35) Brown, M., et al., "Detection of water ice on Nereid," The Astrophysical Journal; 508, L175 to L176, December $1,1998$. 
36) Kevin McPherson, Eric Kelly, Jennifer Keller, ACCELERATION ENVIRONMENT OF THE INTERNATIONAL SPACE STATION, AIAA-2009-0957, 2009.

37) J. GREEN, W. PILAND, "Impact of artificial gravity on habitability for space base," AIAA 7th Annual Meeting and Technical Display, AIAA 1970-1329, 1970.

38) R. A. WENGLARZ, "Problems in altitude control of artificial 'G' space stations with mass unbalance," Journal of Spacecraft and Rockets, 1970, Vol.7: pp. 1161-1166.

39) Jones, R. (editor), “SP-100 planetary mission-system preliminary design study Final report, technical information report," Jet Propulsion Laboratory, JPL D-2513, 1986, 